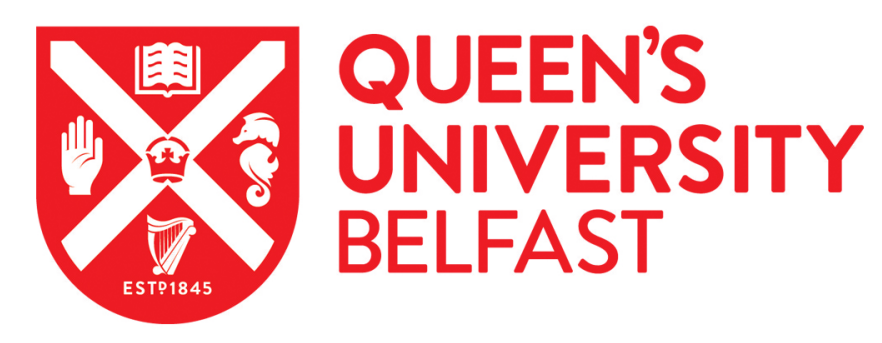

\title{
The Wigner Transition in Nanowires
}

Ballone, P., Hughes, D., \& Cortes-Huerto, R. (2010). The Wigner Transition in Nanowires. In K. D. Sattler (Ed.), Handbook of Nanophysics (pp. 27-1-27-20). Taylor and Francis.

\section{Published in:}

Handbook of Nanophysics

\section{Queen's University Belfast - Research Portal:}

Link to publication record in Queen's University Belfast Research Portal

\section{General rights}

Copyright for the publications made accessible via the Queen's University Belfast Research Portal is retained by the author(s) and / or other copyright owners and it is a condition of accessing these publications that users recognise and abide by the legal requirements associated with these rights.

Take down policy

The Research Portal is Queen's institutional repository that provides access to Queen's research output. Every effort has been made to ensure that content in the Research Portal does not infringe any person's rights, or applicable UK laws. If you discover content in the Research Portal that you believe breaches copyright or violates any law, please contact openaccess@qub.ac.uk. 


\section{7}

\section{The Wigner Transition in Nanowires}

Fermi- versus Luttinger-Liquid Behavior - Spontaneous Polarization of the Electron Gas in 3D, 2D, and 1D • Wigner Crystallization in Q1D Systems • Other Phases

27.3 Fabrication Techniques....

Conducting Polymer Chains • Quantum Point Contacts • Direct Measurements

of Wave Function Localization • Carbon Nanotubes

27.4 Overview of Theoretical Studies.

Idealized Models of Nanowires • Explicitly Correlated Methods • Electron Excitations and Dynamics

27.5 The Jellium Model of Metal Wires and the Density Functional Picture...

27.6 Axially Symmetric Solutions ...27-9

D. Hughes Computations in the Radial-Cylindrical Approach

Queen's University Belfast

27.7 Iterative Minimization of the LSD Density Functional.

27-10

\section{R. Cortes-Huerto}

Queen's University Belfast

Pietro Ballone

Queen's University Belfast

\subsection{Introduction}

Novel techniques to prepare and manipulate systems of nanometric size are being proposed and tested at an amazing and still quickening pace, holding the promise for a revolution in the technological basis of our economy and of our society (Roco et al. 2000). Nanotechnologies already are promoting major new steps in the miniaturization of electronic devices (Cui and Lieber 2001), introducing new concepts in medicine (Loo et al. 2004), changing well-established industrial processes (Zhou et al. 2003), and providing an ever-expanding variety of new ingredients for the next generation of nanostructured materials. In alliance with biotechnologies, nanotechnologies are set to be prominent players in the development of new energy sources and power generation techniques, as well as in the fight to control pollution of the natural environment.

From a purely intellectual perspective, nanotechnologies additionally hold the promise of a wide variety of fascinating new systems and exotic phenomena to explore and to understand. In this respect, nothing can be more intriguing than the electronic properties of nanomaterials, when low dimensionality, quantum mechanics, and drastic size effects combine together to produce unforeseen and counterintuitive effects. Examples of qualitatively new behaviors discovered in nanomaterials include the prominent role of magic sizes in the stability and electronic properties of nanoclusters (de Heer 1993), novel manifestations of superconductivity in nanostructured materials (Schuller et al. 2008), and the super-paramagnetic behavior observed in ferromagnetic nanoparticles (Billas et al. 1993).

Even within this collection of exotic systems and surprising phenomena, the properties of a special class of nanosystems, i.e., nanowires, stand out as particularly remarkable. In the last few years, nanometric conductors have been created by a variety of means, and investigated by experimental techniques ranging from photoemission spectroscopy (Zanolli et al. 2007) to transport measurements (Cao et al. 2005). Examples of the fabrication methods include deposition from the vapor phase and the selective doping of semiconducting nanostructures (Werner et al. 2006), the controlled doping of conducting polymers (Rahman et al. 2006, Rahman and Sanyal 2007), and the etching and mechanical thinning of metal wires. Finally, the fabrication of long and thin nanowires has reached macroscopic production rates with carbon nanotubes (CNTs), representing a wide research field by themselves (Charlier et al. 2007). Systems 
of this kind are bound to play an important role in every future application of nanosystems in electronic devices, since every active element will still need to be connected by a conducting nanowire to become part of a larger functional entity (Beckman et al. 2005).

For all these systems, the first and simplest characterization of their properties may be represented by the determination of their DC electric conductivity, sometimes carried out on individual wires by near-field (scanning tunneling microscopy [STM] or atomic force microscopy [AFM]) microscopy, or measured on mesoscopic samples consisting of many aligned elongated conductors. The electric conductivity of nanowires appears to be quantized in units of $G_{0}=2 e^{2} / h$, that can be explained by scattering theory and by the Landauer theory of conductivity (Landauer 1970). Anomalous values of quantization, however, have been revealed in experiments, pointing to the spontaneous polarization of electrons in a near-1D confining potential (Thomas et al. 1996, Reilly et al. 2002, Bird and Ochiai 2004, Yoon et al. 2007, Danneau et al. 2008).

From a theoretical point of view, the interest in nanowires has been greatly enhanced by the early prediction of new exotic electron states in 1D metals, epitomized by the Tomonaga-Luttinger state (Tomonaga 1950, Luttinger 1963), more commonly referred to as the Luttinger-liquid (LL) phase. Recent experiments are increasingly providing support for the existence of these phases (Bockrath et al. 1999, Zaitsev-Zotov et al. 2000), especially for the thinnest and least defective nanowire samples.

In another manifestation of electron correlation and prominent quantum mechanical behavior, the transition from metallike conduction by delocalized electrons to the insulating behavior of localized electrons has been revealed by experiments in lightly doped polymers (Rahman and Sanyal 2007). Needless to say, any new phenomena affecting conductivity have the potential for a major impact on the prospect of the future miniaturization of electronic components at the nanometric scale.

In $1 \mathrm{D}$, as in higher dimensions, localization is primarily driven by a decrease of carrier density, which enhances the role of correlation with respect to kinetic energy. The original prediction of localization in the context of the homogeneous electron gas is due to Wigner (Wigner 1934). The only parameter relevant to describe the state of this model system is the electron density, $\rho$, or, equivalently, the Wigner-Seitz parameter, $r_{s}=\left(3 / 4 \pi \rho_{\mathrm{b}}\right)^{1 / 3}$ (in $3 \mathrm{D})$, which measures the radius of the sphere that contains, on average, one electron. In the high-density limit, kinetic energy dominates over all other energy contributions (exchange and correlation), the ground state density is translationally invariant, with electrons behaving as independent particles whose wave function, in a constant potential, is a plane wave. Simple quantum mechanical considerations show that decreasing density changes the balance between potential energy (PE) and kinetic energy (KE) contributions, their ratio $\mathrm{PE} / \mathrm{KE}$ being proportional to the Wigner-Seitz radius, $r_{s}$. At sufficiently low density (high $r_{\mathrm{s}}$ ), the system reverts from the homogeneous to a broken-symmetry state in which electrons decrease their potential energy by localization, giving rise to a body-centered cubic lattice (bcc).

By and large, the qualitative picture outlined above for the 3D homogeneous electron gas is also valid in $1 \mathrm{D}$, even though quantitative details need to be adjusted according to the space dimension. To be precise, the relative role of fluctuations increases with decreasing dimensionality and, in $1 \mathrm{D}$, long-range periodicity is prevented by the divergence of the mean square fluctuation in the particles' position (Peierls instability). This last statement, however, is strictly valid only in the thermodynamic limit, i.e., for infinitely extended systems. Real systems, instead, are always finite, implying at the same time that the localization transition cannot be infinitely sharp, and that the role of fluctuations is mitigated with respect to the extended case. As a result, localized configurations persist over mesoscopic and even macroscopic times, and could be observed by experiments. Indeed, several groups have already measured phenomena that strongly point toward the formation of a Wigner crystal (WC) in a variety of quasi-1D (Q1D) systems (Hiraki and Kanoda 1998, Horsch et al. 2005, Rahman and Sanyal 2007). In all cases, the Q1D WC (Schulz 1993) is primarily the result of low density, with confinement playing a minor role.

This chapter is focused precisely on the localization (Wigner) transition taking place in Q1D metallic wires with decreasing carrier (electrons or holes) density. In what follows we provide, first of all, a brief overview of the phases believed to occur in Q1D systems. These include the "normal" regime, believed to be $\mathrm{LL}$, which is contrasted to the regular Fermi liquid (FL); a magnetically ordered phase; a low-density, crystalline regime; and finally, other even more exotic phases identified by dynamical or excited-state properties. Moreover, we summarize a number of theoretical studies, ranging from the use of simple idealized models, to state-of-the-art correlated methods.

To emphasize the fact that the low-carrier-density nanowires we are interested in are not simply a theoretical curiosity, we provide a short summary of the experimental methods that have been used to fabricate wires of nanometric diameter over a wide range of carrier densities.

In the second half of the chapter, we introduce an idealized (jellium) model of metal wire, and we investigate the localization transition by a conceptually simple and computational tractable approach based on density functional (DF) theory (Hohenberg and Kohn 1964, Kohn and Sham 1965). The approximation we use (local spin density, LSD) to treat exchange and correlation has been devised for electron systems in a low correlation regime, and the quantitative predictions of the method need to be taken with some caution. However, the method and the results it provides have a great didactical value, since they describe the system and its localization transition in simple and intuitive terms. According to the results of our DF computations, the localization transition is intrinsically related to the spontaneous spin polarization expected in low-density electron systems. For this reason, we also briefly cover this transition in our discussion. 


\subsection{The Phase Diagram of the Q1D Electron Gas}

\subsubsection{Fermi- versus Luttinger-Liquid Behavior}

The FL picture (Giuliani and Vignale 2005) of two- and threedimensional systems explains why the electron-electron (ee) interaction between particles close to the Fermi surface may be neglected, therefore justifying the apparent success of the independent particle, or free-electron gas description of such systems. Only at low charge carrier densities does the role of $e e$ interaction begin to manifest itself, causing a breakdown in the quasi-particle description. Such systems are referred to as correlated and constitute "the exception rather than the rule." For 1D systems, the dimensional constraints give rise to enhanced correlation at all densities, rendering the quasi-particle formalism useless. In 1950, Tomonaga (1950) proposed a simplified model of the $1 \mathrm{D}$ electron gas, that was later generalized by Luttinger (1963) in 1963. It has subsequently been revised by several other authors (see Schulz et al. 2000, and references therein). These models provided a qualitatively different understanding of the behavior of $1 \mathrm{D}$ systems.

As it is also the case for the familiar FL model, Tomonaga and Luttinger considered the behavior of electrons whose energy is close to the chemical potential (or Fermi energy). Outside the range of energies considered, states are assumed not to be influenced as strongly by perturbations in the potential. The details of the Luttinger model are not presented here, though its consequences shall be discussed. A review article by Schulz et al. (2000) provides greater depth.

The solution of the Luttinger Hamiltonian is a nontrivial exercise and may not be achieved by perturbative methods. Rather, it is necessary to replace the field operators in the second quantized Hamiltonian (Inkson 1984, Negele and Orland 1998) with a new set of operators, which, upon inspection, are seen to fulfill the commutation relations of boson operators. It then becomes apparent that the low energy collective excitations of such systems behave as a set of massless bosons. Moreover, the Hamiltonian may be factorized into separate charge (C) and spin (S) components, $\hat{\mathcal{H}}=\hat{\mathcal{H}}_{\mathrm{C}}+\hat{\mathcal{H}}_{\mathrm{S}}$. This gives rise to a phenomenon known as spin-charge separation. Supposing an electron is injected into the system at a given time and position endowing it with a net spin and charge equivalent to one electron, at some later time the spin and charge will be spatially separated. This is not to say that the electron has in some way split, but rather, the system has entered an excited state, forming two collective excitations: a holon (charge quanta) and a spinon (spin quanta). A different variety of LL, also known as the spin-incoherent LL (Fiete 2007), has recently attracted considerable attention. This phase occurs when the energy scale associated with charge degrees of freedom is vastly larger than the energy scale of spin excitations.

Another interesting consequence of the Luttinger model is that the presence of even the smallest impurity will give rise to an insulating state at low temperatures. Linear response theory reveals that in the ground state the system is on the verge of forming a charge density wave (CDW) of wavelength $2 k_{F}$. Introducing an impurity is sufficient to "pin" this wave in position, thereby reducing the conductivity to zero. This situation is often referred to as a dirty LL (Ogata and Fukuyama 1994). If the temperature of the system or the bias across the system is increased above a given threshold, the system enters the conducting phase again. Experimentalists look for specific, power law dependence of the conductivity on temperature $G \propto T^{\alpha}$, and of the differential conductance on the applied bias $\mathrm{d} I / \mathrm{d} V \propto T^{\alpha+1}$, as an indication of LL behavior (Giuliani and Vignale 2005). This property of 1D systems makes experimental investigation difficult, as only highly pure samples will exhibit true LL behavior. Moreover, experimentally distinguishing between the pinned CDW and WC formation presents difficulties (Horsch et al. 2005).

LL behavior is only observed in a small number of cases (Zaitsev-Zotov et al. 2000) though it is believed that it provides a more accurate description of Q1D systems than the FL formalism. Examples include, CNTs (Bockrath et al. 1999), and some less-common classes of organic (Biermann et al. 2002) and inorganic (Horsch et al. 2005) conducting polymers. Recently, it has been suggested that electrons at an armchair edge of an undoped 2D carbon plane (graphene) might exhibit LL properties (Fertig and Brey 2006).

\subsubsection{Spontaneous Polarization of the Electron Gas in 3D, 2D, and 1D}

At sufficiently low densities, the ground state of the 3D electron gas is known to become ferromagnetic (Ceperley and Alder 1980). Since the kinetic and exchange energies scale as $r_{\mathrm{s}}^{-2}$ and $r_{\mathrm{s}}^{-1}$, respectively (Mahan 2000, Giuliani and Vignale 2005), it is clear that for large $r_{\mathrm{s}}$, the exchange energy dominates and the system will polarize. Hartree-Fock calculations predict the polarization transition to occur at $r_{\mathrm{s}}=5.45$. However, the inclusion of correlation energy destabilizes the spin-polarized state, moving the transition to lower densities (Ceperley and Alder 1980). One recent study finds the polarization phase transition to occur over a continuous range of densities, $20 \pm 5<r_{\mathrm{s}}<40$, where above $r_{\mathrm{s}}=40$ the system is fully polarized (Ortiz et al. 1999). The precise location of this phase transition, however, is still a matter of debate (Zong et al. 2002).

The effect of confining a system in two dimensions (or indeed one or zero dimensions) is to enhance the role of exchange and correlation energy (Zabala et al. 1998). It is therefore believed that the polarization state is stabilized, and the corresponding value of transition density is increased. Several groups have measured anomalous values of the conductance in quantum point contacts (QPC) (Thomas et al. 1996, Reilly et al. 2002, Bird and Ochiai 2004, Yoon et al. 2007, Danneau et al. 2008), which is believed to point toward the existence of a partially polarized ground state. 


\subsubsection{Wigner Crystallization in Q1D Systems}

In 1934, Wigner (Wigner 1934) proposed that at sufficiently low densities, when $e e$ interaction dominates over electron kinetic energy, electrons could attain a more stable state through localizing in space. Rather than being spread out in Bloch waves, electrons would reside in well-defined, localized regions, forming a bcc lattice. In doing so, a cost is paid in terms of kinetic energy; however, the resultant gain in correlation energy makes this phase preferable.

The WC was first observed experimentally in 1979 within a layer (2D) of liquid helium (Grimes and Adams 1979). It has subsequently been observed in several Q1D systems as we shall outline below (Hiraki and Kanoda 1998, Horsch et al. 2005, Rahman and Sanyal 2007).

The WC has been studied intensively by a variety of methods, including Hartree-Fock methods (Trail et al. 2003), which drastically overestimate its stability. Several DF computations, whose energy functionals are based on data gathered from QMC simulations, have been used to study this phase transition (Senatore and Pastore 1990, Ito and Teraoka 2004, 2006). Benchmark diffusion, fixed-node quantum Monte-Carlo simulation place the WC transition in the region $65 \pm 10<r_{\mathrm{s}}<106$ (Ceperley and Alder 1980, Ortiz et al. 1999, Drummond et al. 2004).

Finally, the properties of a zigzag variety of WC have been reviewed by Meyer and Matveev (2009).

\subsubsection{Other Phases}

For completeness we briefly mention some additional phases observed in Q1D systems.

Mott insulators have been observed in organic nanowires, generated using nanoscale-electrocrystallization methods (Hasegawa et al. 2008), and also in single-crystal $\mathrm{VO}_{2}$ nanowires, grown using physical vapor deposition methods (Baik et al. 2008). The transition to the Mott insulator has been investigated using many-body Green's function methods (Biermann et al. 2001).

The Bechgaard salts, a class of organic charge-transfer complexes, provide an example of a Q1D system that exhibits high temperature superconductivity (Biermann et al. 2002). Nickel et al. (2006) have used a renormalization group approach to investigate the phase diagram in Q1D systems, and have focused in detail on the superconducting phase.

\subsection{Fabrication Techniques}

A comprehensive summary of fabrication techniques for nanometric wires is well beyond the scope of this chapter. We provide here only a brief overview of the most common techniques for nanowire growth, focusing in particular on the methods that have been used to fabricate wires operating in the low-carrierdensity regime, or high correlation limit.

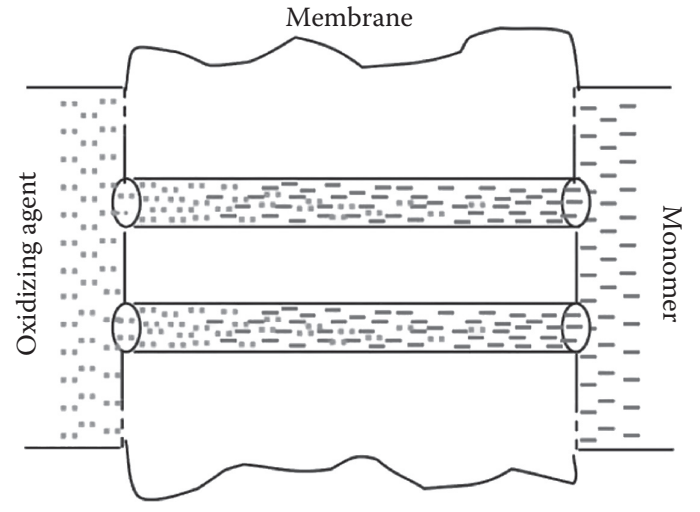

FIGURE 27.1 Schematic drawing of the experimental setup used to prepare conducting polypyrrole nanowires in porous membranes. (From Rahman, A. et al., Phys. Rev. B, 73, 125313, 2006. With permission.)

\subsubsection{Conducting Polymer Chains}

The channels of porous polycarbonate membranes have been used as a template for the growth of monodisperse, parallel bundles of polypyrrole nanowires (see Figure 27.1). The carrier concentration within such systems may be varied over a wide range by doping (Rahman and Sanyal 2007). In the procedure described in (Parthasarathy and Martin 1994), for instance, a polycarbonate membrane was immersed in a refrigerated solution containing the monomer pyrrole ring. Polymerization occurs in the pores of the membrane and over the surface. Surface polymers are removed by polishing techniques. If desired, it is possible to isolate individual wires by dissolving the membrane in dichloromethane; wires are then removed from solution by filtering. Electrical properties of the wires are measured by coating opposite surfaces (at either end of the wires) with gold, which has low electrical resistance. Both gold surfaces may then be connected to a current or voltage source and the electrical properties of many parallel wires measured and averaged over. Scanning electron microscopy (SEM) and tunneling electron microscopy (TEM) verify that polypyrrole wires are unbroken, and are not hollow, which would lead to erroneous measurements. Both of these failures plagued prior attempts to synthesize conducting polymer wires from polyaniline (Parthasarathy and Martin 1994).

Measurements of conductance properties, point to a strong temperature and bias dependence (Rahman and Sanyal 2007), findings that would be consistent with the formation of a WC.

\subsubsection{Quantum Point Contacts}

QPC represent arguably the simplest way to create nanowire structures. Several fabrication methods have been developed. These include wires created by contact between atomic force microscopes (AFM) and an underlying surface (Kuipers and Frenken 1993) (Figure 27.2). In such a case, the AFM is brought close to a surface, at which point it is speculated that van der Waals interactions give rise to a point contact. 


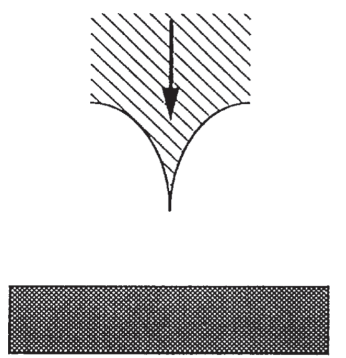

(a)

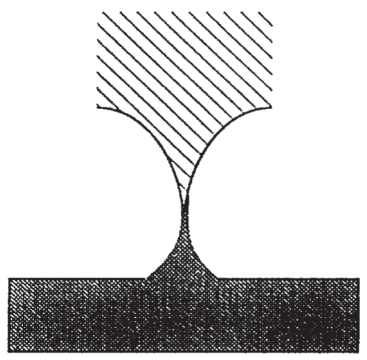

(b)

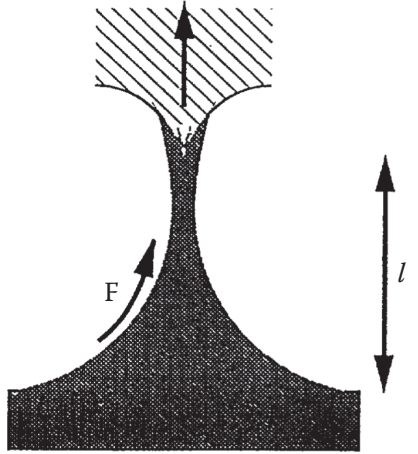

(c)

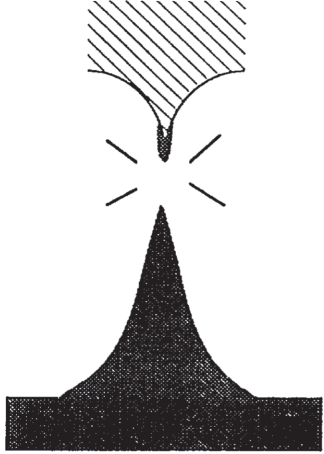

(d)

FIGURE 27.2 Schematic cycle of the formation and rupture of a surface-tip contact: (a) Approach of the tip. (b) Just after the jump to contact. (c) Growth of neck. (d) Breaking of the neck. (From Kuipers, L. and Frenken, J.W.M., Phys. Rev. Lett., 70, 3907, 1993. With permission.)

Contacts created in this way have a low aspect ratio (radius/ length), and translational invariance may not be assumed. Moreover, the range over which electron densities may be controlled, in either class of QPC, is very narrow, and is fundamentally limited by the material used.

A second type of QPC is commonly assembled using molecular beam epitaxy (MBE) (Werner et al. 2006), a form of thin film deposition (Fan et al. 2006). Current is free to flow through a GaAs 2D electron gas (2DEG) connecting two ohmic contacts (Figure 27.3). By increasing the negative bias to a set of gates, which are separated from the 2DEG by an insulating AlGaAs layer, the effective width of the channel through which electrons are free to move is progressively reduced. Recent experiments of Bird et al. (Bird and Ochiai 2004, Yoon et al. 2007) have measured anomalous conductances of a point contact fabricated using such techniques, pointing to spontaneous spin polarization in high density samples. This phenomenon, commonly referred to as the " 0.7 anomaly", was first measured by Thomas et al. (1996), and its origin is still the subject of debate (Danneau et al. 2008). Other experiments have investigated the dependence of this anomaly on the electron density (Reilly et al. 2002).

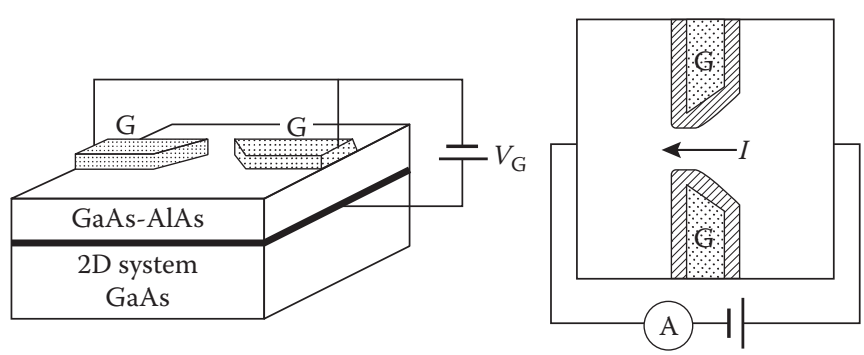

FIGURE 27.3 Schematic cross-sectional, and top view of a quantum point contact, defined in a high-mobility $2 \mathrm{D}$ electron gas at the interface of a GaAs/AlGaAs heterojunction. The point contact is formed when a negative voltage is applied to the gate electrodes on top of the AlGaAs layer. Transport measurements are made by employing contacts to the $2 \mathrm{D}$ electron gas at either side of the constriction. (From Kawabata, A., Rep. Prog. Phys., 70, 219, 2007. With permission.)

\subsubsection{Direct Measurements of Wave Function Localization}

Auslaender et al. (Auslaender et al. 2002, 2005, Steinberg et al. 2006) have produced a novel, thin film based experimental setup, comprising two parallel wires embedded in a GaAs/AlGaAs heterostructure (Figure 27.4). This has been used to gain direct insight into the possible localization of the many-body wave function.

The device is formed using a method known as cleaved-edge overgrowth (Pfeiffer et al. 1990), a type of MBE. The setup uses a technique known as momentum-resolved tunneling spectroscopy to measure the tunneling current between two parallel quantum wires, and thereby build a picture of the many-body wave function of the upper wire (UW). It is possible to control the energy and density of the electrons in the UW by altering bias $V_{\mathrm{SD}}$ and $V_{\mathrm{G}}$, respectively. A magnetic field $B$, applied in a direction perpendicular to the wires' plane, supplies the electrons with momentum $k_{\mathrm{B}}=e B d / \hbar$ allowing tunneling to the longer of the two wires, or lower wire (LW). Knowledge of tunneling conductance $G\left(V_{\mathrm{SD}}, B\right)$ as a function of magnetic field strength, $B$, and bias $V_{\mathrm{SD}}$ is then related to the square of the many-body wave function in momentum space.

Not only has evidence of spin-charge separation been measured, pointing toward LL behavior (Auslaender et al. 2005), but when electron density in the UW has been depleted sufficiently, the wave function is seen clearly to localize, pointing toward the formation of a WC (Steinberg et al. 2006).

Mueller (2005) has modeled the system in three different regimes: the high-density regime, using a Thomas-Fermi description; the low-density regime, using semiclassical arguments; and in an attempt to understand the processes occurring during localization, a Hartree-Fock model was implemented. The models implemented are purely $1 \mathrm{D}$, making it necessary to approximate the interelectron repulsion as follows: $U(r)=$ $\min \left(|r|^{-1}, d^{-1}\right)$, where $r$ is the ee separation distance, and $d$ is a parameter. Tunneling between wires is treated in lowest order perturbation theory, which the author identifies as a significant source of error. Moreover, the tunneling current is found 


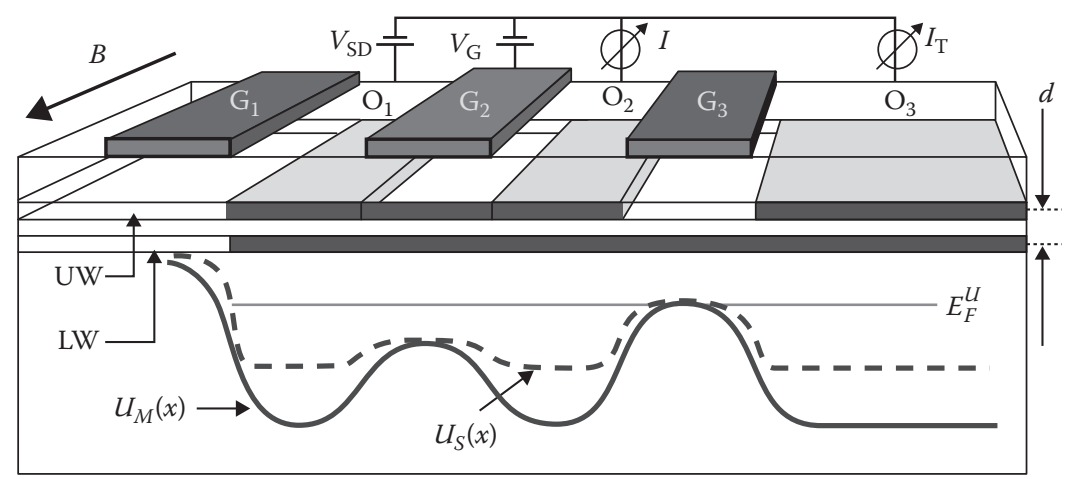

FIGURE 27.4 Schematic drawing of tunneling spectrometer. From top to bottom: Magnetic field, $B$, perpendicular to the plane of the wires. $2 \mu \mathrm{m}$ wide gates $\left(\mathrm{G}_{1}, \mathrm{G}_{2}\right.$, and $\left.\mathrm{G}_{3}\right)$ and Ohmic contacts $\left(\mathrm{O}_{1}\right.$; source, and $\mathrm{O}_{2,3}$; drains). Gate voltage, $V_{\mathrm{G}}$, serves as an electron-density controller for the upper wire (UW), two-terminal current $I$, and $I_{\mathrm{T}}$ tunneling current. $20 \mathrm{~nm}$ thick $\mathrm{UW}$ at the edge of the $2 \mathrm{D}$ electron gas, $6 \mathrm{~nm}$ insulating AlGaAs barrier, and $30 \mathrm{~nm}$ thick lower wire (LW). Single mode (and multimode) potentials $U_{S}(x)\left(U_{M}(x)\right)$ experienced by UW. (From Steinberg, H. et al., Phys. Rev. $B, 73,113307,2006$. With permission.)

to be lowered by the use of the Hartree-Fock approximation, which additionally stabilizes the WC phase. Nevertheless, the method provides a qualitative view of the transition between a smooth density profile, which gradually develops Friedel oscillations, that become so marked that Wigner crystallization finally occurs.

Bird and collaborators have reported a similar experimental setup of two parallel wires, in which the effective width of one wire is varied. The conductance of the second wire is measured, which exhibits a resonant peak close to the pinch-off of the first wire (Morimoto et al. 2003). The origin of this effect remains unexplained, however, points to the existence of correlation between the two systems.

\subsubsection{Carbon Nanotubes}

CNTs consist of a graphene sheet rolled up to form a cylinder. CNTs can be generated with extremely large aspect ratios. Moreover, it is possible to form more exotic structures containing Y- and X-shaped junctions and budded structures. Also, nanotubes may be arranged coaxially (multi-walled CNTs) or in parallel bundles. It is possible to alter the chirality of the tube, specified by the graphene lattice vector: this affects electrical and optical properties. Dopant levels and electrical properties of CNTs may be controlled in such a way as to achieve metallic or semiconducting behaviors. For a review on most aspects of CNTs see Charlier et al. (2007). Moreover, by intercalating various types of nanotubes (for example, carbon, $\mathrm{BN}, \mathrm{MgO}, \mathrm{Ga}_{2} \mathrm{O}_{3}$ ) with other materials, very long wires, with highly varied electrical properties (Kim et al. 2007, Zanolli et al. 2007, Gong et al. 2008) have been created.

Cao et al. (2005) have successfully grown CNTs between tungsten/platinum electrodes suspended over a metal electrode (see Figure 27.5). Altering the potential of the electrode (gap voltage) enhances or reduces the active charge carrier concentration, modifying the electrical behavior of the nanowire. CNTs may be generated with an extremely low number of defects and impurities, a quality that is essential for the study of $1 \mathrm{D}$ systems.

Techniques for generating nanowires using CNTs are widely different, but chemical vapor deposition is one of the most popular (José-Yacamán et al. 1993).
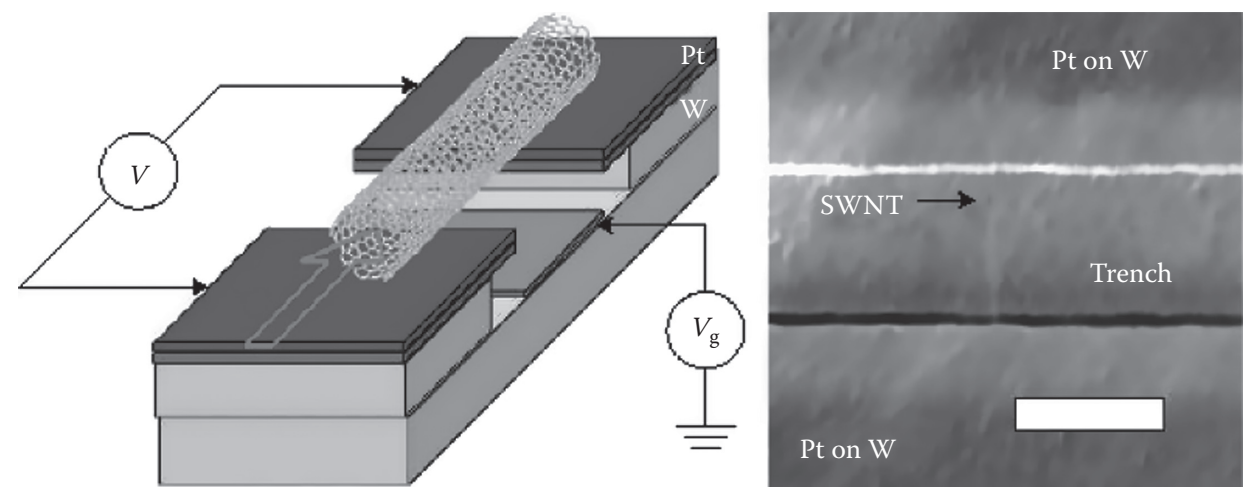

AQ1 FIGURE 27.5 Left: Schematic drawing of a carbon nanotube (CNT) bridging two platinum electrodes. The CNT spans a trench containing a gate used to vary charge carrier concentration. Right: SEM image of the experimental setup. (From Cao, J. et al., Nature, 4, 745, 2005. With permission.) 


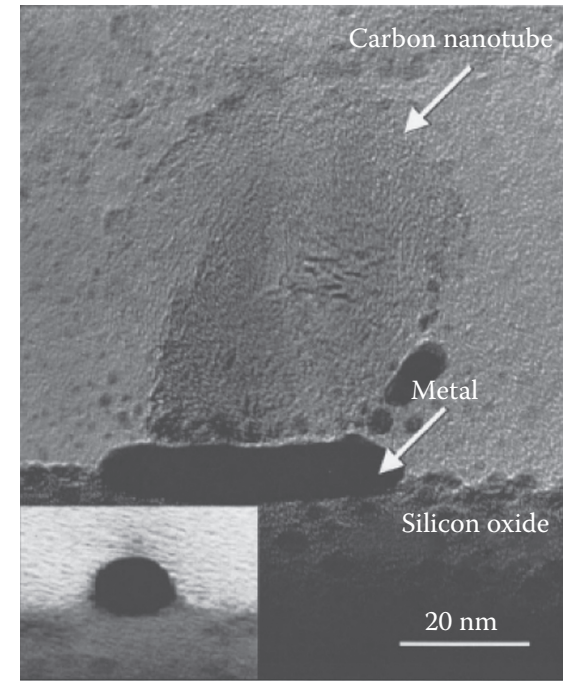

(a)

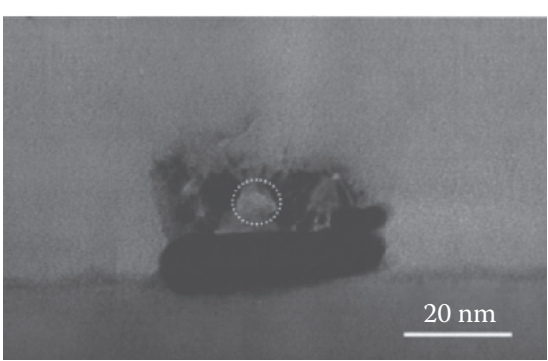

(b)

FIGURE 27.6 Cross-sectional TEM images. (a) A $50 \mathrm{~nm}$ wide Au/Ti nanowire was formed underneath a CNT of about $60 \mathrm{~nm}$ in diameter. Inset: A Au/Ti nanowire of $12 \mathrm{~nm}$ in width is shown. (b) A metal nanowire of $40 \mathrm{~nm}$ in width was formed below a bundle of CNTs. White circle encloses one of the CNTs. (From Yun, W.S. et al., J. Vac. Sci. Technol. A, 18, 1329, 2000. With permission.)

In most cases, these structures have been seen to behave as FL; however, several studies have detected indications of LL behavior (Bockrath et al. 1999), and one more recent study points toward the formation of a 1D WC (Deshpande and Bockrath 2008).

In addition to acting as conducting wires themselves, CNTs have been used as masks for the etching of $\mathrm{Si}$ substrates (Yun et al. 2000) (Figure 27.6). With advances in CNT manipulation technologies (Hertel et al. 1998) the authors identify this as a realistic option for future, large-scale nanowire fabrication.

\subsection{Overview of Theoretical Studies}

The variety of theoretical methodologies implemented in the study of Q1D systems is truly extensive, ranging from the simplest fully-1D Thomas-Fermi (Tanatar et al. 1998) models, to sophisticated many-body techniques (Biermann et al. 2001). The study of transport properties in nanowires also constitutes a major portion of theoretical effort, this is due to a number of reasons, for example, the need to understand dynamical processes such as heating and power dissipation in structures intended for device applications (Montgomery et al. 2002).

We present a short summary of some of the main areas of recent theoretical focus concerning $1 \mathrm{D}$ and Q1D systems, drawing particular attention to the study of low-density (or highly correlated) systems.

\subsubsection{Idealized Models of Nanowires}

Idealized models play a powerful role in the study of quantum systems. By eliminating irrelevant degrees of freedom, for example, the nuclear degrees of freedom in the case of jellium models, one is free to explore the physics of correlated electronic motion, without added complications generated by interactions with a complex lattice of nuclei.

A number of studies have concentrated on truly 1D models (Tanatar et al. 1998, Mueller 2005, Casula et al. 2006, Shulenburger et al. 2008). In such cases, the Coulomb potential is modified to remove the singularity at zero separation. Typical approximations include $U(r)=\min \left(|r|^{-1}, d^{-1}\right)$ or $U(r)=\left(d^{2}+\right.$ $\left.r^{2}\right)^{-1 / 2}$, where $r$ is the interelectron separation, and $d$ is a small, finite parameter.

As mentioned above, Mueller (2005) has investigated one such model. A combination of Thomas-Fermi, Hartree-Fock, and semiclassical techniques, in parallel with perturbation theory, have been used to gain insight into the observation of charge localization, and the possibility of Wigner crystallization in the tunneling spectroscopy experiments of Auslaender et al. (2002). This study provides a qualitative view of the transition of the electron density between regions of high charge density to low charge density. In doing so, crystallization is directly observed.

Tanatar et al. (1998) have used a 1D Thomas-Fermi approach to investigate the transition to the localized density regime for purely $1 \mathrm{D}$ wires. One additional level of approximation is introduced, in that the form of the density is assumed to have a cosine modulation. The results point to a Wigner transition at a density that appears to overestimate the one observed in experiment. The influence of introducing a second parallel wire has also been investigated: this additional interaction further stabilized the crystal phase.

Several authors have used more realistic Q1D models, often based on the jellium picture, which facilitate the treatment of much larger wire radii (Yannouleas and Landman 1997, Zabala et al. 1998). Several reviews (Brack 1993, de Heer 1993) highlight the success that the jellium model has enjoyed when applied to the valence electrons in simple metal clusters. One 
might therefore expect a comparable degree of success when applying a similar jellium model to nanowires. For brevity we do not elaborate on the specific details of these models, other than to say that the methods used vary greatly, from the simplest free-electron gas picture (Stafford et al. 1997), to modified Thomas-Fermi approaches (Yannouleas and Landman 1997, Yannouleas et al. 1998), and finally to DF-based simulations, in the local-density approximation (Perdew et al. 1990, Zabala et al. 1998, 1999). Also, the geometries used vary from infinitely extended, and having cylindrical cross-sections (Zabala et al. 1998, 1999), to finite systems (Yannouleas and Landman 1997, van Ruitenbeek et al. 1997), some of which have constrictions, or necks, introduced in the axial direction (Stafford et al. 1997, Yannouleas et al. 1998).

Such models (Yannouleas and Landman 1997, Yannouleas et al. 1998, van Ruitenbeek et al. 1997, Stafford et al. 1997) have been used to partially explain the experimentally observed oscillations in elongation forces, and conductivities, when a wire is subjected to strain. In addition to the abrupt changes in atomic position that occur when a wire is stretched, the mean radius of the wire will be reduced also (provided one is assuming a fixed volume). It is argued that for a given background density, parameterized by the Wigner-Seitz radius, $r_{\mathrm{s}}$, certain critical values of the wire radius result in particularly stable electronic configurations, known as magic radii. The enhanced stability, produces peaks in the elongation force, defined as $F=-\mathrm{d} E / \mathrm{d} L$, where $E$ is the energy of the wire of radius $R_{\mathrm{B}}$ and $L$ is its length. Similar arguments reveal oscillations in the conductance as a function of radius.

Zabala et al. $(1998,1999)$ has used an infinitely extended, Q1D jellium model to investigate the existence of stable, partially polarized ground states in systems within metallic range of densities $\left(2 \leq r_{\mathrm{s}} \leq 6\right)$. The net polarization is small, only amounting to a fraction of a Bohr magneton, $\mu_{\mathrm{B}}$, per atomic unit of length, $a_{0}=\hbar / m_{e} e^{2}$. An explanation, of such polarized states was provided using the Stoner criterion.

To the best of our knowledge, such Q1D models have not been used to investigate the crystalline or low-density phases of the confined electron gas.

\subsubsection{Explicitly Correlated Methods}

We have already emphasized that 1D systems experience enhanced $e e$ correlation regardless of the charge carrier concentrations, resulting directly from the dimensional constraints. It is widely accepted that mean-field descriptions, for instance DF, are insufficiently accurate to provide a fully quantitative description of such correlated materials (Kotliar and Vollhardt 2004), and in several cases, even the qualitative picture provided by DF might be incorrect.

Dynamical mean-field theory (DMFT) was developed specifically for the treatment of correlated materials (Kotliar et al. 2006), based on a lattice representation of the system. In short, the theory maps any lattice to a single-site problem, which interacts with a self-consistent bath, or continuum of parameterized, noninteracting excitations. Particles are free to enter and leave the site from the continuum, and vice-versa, which contains a number of occupied and unoccupied states. This hybridization with the continuum leads to the inclusion of dynamic or frequency-dependent properties. The resulting equations are typically solved by Monte-Carlo methods.

Chain-DMFT was developed specifically for the treatment of Q1D materials (Arrigoni 1999, 2000, Georges et al. 2000). In this case, particles are free to "hop" along individual chains, represented by a single site coupled to a self-consistent bath. However, it is possible to include perpendicular hopping between chains, in which case we recover DMFT. Biermann et al. (2001) have used chain-DMFT to investigate the de-confinement transition. That is, the transition between Mott-insulator (MI), to LL, to FL behavior. They have successfully identified the transition between LL and FL regimes as a function of lowering temperature. Moreover, the transition between MI and FL has been simulated by increasing the effective width of the chain, that is, by introducing perpendicular hopping. Deconfinement behavior has been realized experimentally in the Bechgaard salts (Biermann et al. 2002).

A renormalization group (Fisher 1998) approach has been taken by Nickel et al. (2006) in attempting to quantify the phase diagram of Q1D materials, placing particular focus on the superconducting phase. The formation mechanisms behind, and behavior of collective excitations, such as, CDW and spin density waves (SDW) has been explored in depth.

Casula et al. has performed benchmark, lattice-regularized, diffusion Monte-Carlo simulations of a Q1D electron gas, assuming a harmonic confining potential in the radial direction (Casula et al. 2006). The study has been used to parameterize a correlation functional, and has successfully observed the formation of a WC at low densities. Results are found to be consistent with the predictions of the Luttinger model.

Yang et al. have performed state-of-the-art Bethe-Salpeter and GW calculations of the optical spectra of Si nanowires of diameter in the range 1-2 $\mathrm{nm}$ (Yang et al. 2007). They find strong deviations in the spectra when compared to bulk silicon that are attributed to dimensional confinement effects. Several other GW-based investigations point to similar effects (Zhao et al. 2004, Yan et al. 2007).

\subsubsection{Electron Excitations and Dynamics}

Almost all experimental investigations into nanowire behavior and classification rely on measuring transmission currents, conductances, and differential conductances through the sample of interest (Rahman and Sanyal 2007, Steinberg et al. 2006, Bockrath et al. 1999, Deshpande and Bockrath 2008). Although, other techniques are commonly used, such as photoluminescence emission spectroscopy (Zanolli et al. 2007), transport measurements dominate. We therefore emphasize the importance of studying the dynamical and transport properties of nanowires. Moreover, the study of nanowires plays an essential role in the future of nanoelectronics and device miniaturization. 
Landauer theory (Landauer 1970) predicts quantization of the conductance $G=\left(2 e^{2} / h\right) T K$, where the interaction parameter $K=1$ for noninteracting systems, $K<1$ for repulsive interactions, and $K>1$ for attractive interactions. Also, $T$ is the transmission probability, often assumed to have value of unity.

As we saw above, recent experiments (Thomas et al. 1996, Reilly et al. 2002, Bird and Ochiai 2004, Yoon et al. 2007, Danneau et al. 2008) have observed intermediate plateaus in the conductance, in the range $0.5-0.7 \times 2 e^{2} / h$. Such deviations from the traditional Landauer picture have been explained by the formation of spin moments, arising due to confinement.

A recent review paper by Kawabata (2007) summarizes many of the standard features of modern transport simulations. We also highlight the work of Todorov and his group, who have performed a number of time-dependent tightbinding simulations, focusing on the effects of heating and power dissipation due to current flow in nanometric wires (Montgomery et al. 2002). Indeed, transport computations constitute a large portion of all modern literature concerning nanowires.

As we have indicated previously, our primary area of interest is the low charge density regime. In this respect, we highlight the work of Lenac (2005), who has modeled the interaction of the phonons of a 2D WC with the electromagnetic field. This work has been used to model dispersion relations for polaritions and riplons, that might be observed experimentally, for example, on the surface of liquid He (Grimes and Adams 1979).

Gold et al. have used generalized, frequency-dependent linear response computations to model the excitation spectra of CDWs and SDWs in Q1D systems (Gold and Calmels 1998). The effects of varying the background density as well as the polarization of the system have been incorporated.

\subsection{The Jellium Model of Metal Wires and the Density Functional Picture}

A broad view of the different phases and properties relevant for nanometric conducting wires across a wide density range can be obtained by computational investigations based on the DF theory (Hughes and Ballone 2008). The jellium model and the DF methods used in this and in the following section are closely related to general models and computational techniques widely used in condensed matter physics, (Ashcroft and Mermin 1976, de Heer 1993, Brack 1993, Martin 2004) and are briefly described here mainly for completeness and for didactical purposes.

We consider a highly idealized model of nanowires consisting of a rod-like distribution of positive charge, and of $N$ electrons moving in the electrostatic field due to all the charges in the system. The wire segment has length $L$, it is globally neutral, and is oriented along the $z$ direction (see Figure 27.7). Cylindrical coordinates $(r, \phi, z)$ are used throughout the chapter, together with Hartree atomic units.

The simplest model is obtained by considering the following distribution of positive charge:

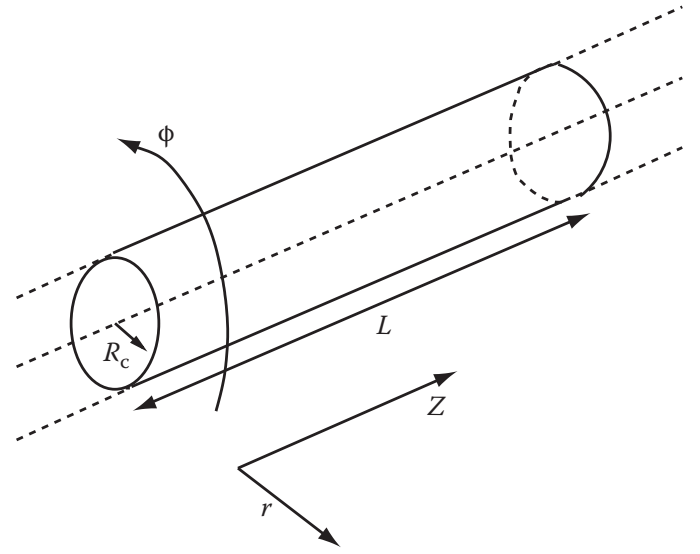

FIGURE 27.7 Schematic drawing of a cylindrical jellium nanowire.

$$
\rho_{+}(\mathbf{r})=\rho_{+}(r, z)= \begin{cases}\rho_{\mathrm{b}} & \text { for } r \leq R_{\mathrm{c}}, 0 \leq z<L \\ 0 & \text { for } r>R_{\mathrm{c}}, 0 \leq z<L\end{cases}
$$

where $R_{\mathrm{c}}$ is the radius of the cylindrical charge density, and, in what follows, $R_{\mathrm{c}} \ll L$. In other words, the positive density is constant and equal to $\rho_{\mathrm{b}}$ within the cylinder, and it vanishes outside. Following the standard practice of electron gas studies (Ashcroft and Mermin 1976), the background density, $\rho_{b}$, is measured by the radius, $r_{\mathrm{s}}$, of the sphere that contains one unit charge, $\left(r_{\mathrm{s}}=\left[3 / 4 \pi \rho_{\mathrm{b}}\right]^{1 / 3}\right)$.

According to the basic results of the DF theory (Hohenberg and Kohn 1964, Kohn and Sham 1965), the ground state energy and density of an A-electron system in an external potential, $V_{\text {ext }}$ (r), can be determined by minimizing a universal functional of the electron density:

$$
\begin{aligned}
E_{\mathrm{KS}} & {\left[\left\{\psi_{i}\right\} ; i=1, \ldots, N\right] } \\
& =\sum_{i=1}^{N}\left\langle\psi_{i}\left|-\frac{1}{2} \nabla^{2}+V_{\mathrm{ext}}(\mathbf{r})+\frac{1}{2} V_{\mathrm{Ha}}(\mathbf{r})+u_{\mathrm{XC}}[\rho]\right| \psi_{i}\right\rangle
\end{aligned}
$$

with respect to the $N$ occupied Kohn-Sham (KS) orbitals $\left\{\psi_{i}\right\}$, that, in turn, determine the electron density according to the relation

$$
\rho(\mathbf{r})=\sum_{i=1}^{N}\left|\psi_{i}(\mathbf{r})\right|^{2}
$$

In Equation 27.2, $V_{\mathrm{Ha}}$ is the electrostatic potential of positive and negative charges, and therefore it includes the contribution of the external field due to the positive background as well as the Hartree potential due to the electron distribution. Moreover, $u_{\mathrm{Xc}}[\rho]$ is the exchange-correlation (XC) energy per electron, accounting for Fermi statistics (exchange) and other many-body effects (correlation). In our computations, the external field is represented by the Coulomb potential of the positive background, and therefore is included into $V_{\mathrm{H}_{\mathrm{a}}}$. Moreover, partly for 
the sake of simplicity, $u_{\mathrm{xc}}[\rho]$ is given by the LSD approximation (Ceperley and Alder 1980).

Application of the Euler-Lagrange theorems to the minimization of the functional (27.2) shows that this problem is equivalent to the self-consistent solution of the Schroedinger-like equations:

$$
\left\{-\frac{1}{2} \nabla^{2}+V_{\mathrm{Ha}}(\mathbf{r})+\mu_{\mathrm{XC}}[\rho]\right\}\left|\psi_{i}\right\rangle=\varepsilon_{i}\left|\psi_{i}\right\rangle
$$

for the $N$ states of lowest eigenvalues. In the equation above, $\mu_{\mathrm{XC}}[\rho]=u_{\mathrm{xC}}[\rho]+\rho \mathrm{d} u_{\mathrm{xC}}[\rho] / \mathrm{d} \rho$ is the XC potential corresponding to the XC energy, $u_{\mathrm{xC}}[\rho]$, of Equation 27.2.

Finite-size effects are minimized by periodically repeating the basic segment in the $z$ direction, thus approaching the limit of a geometric wire extending to infinity along a single direction. The periodicity along $z$ implicitly defines a 1D Brillouin zone (BZ) of width $2 \pi / L$, and electron states can be labeled with a continuous wave vector, $k_{z}$, belonging to the BZ. Results do not depend on the choice of the periodicity $L$ and of the corresponding number $N$ of electrons in the simulation cell for systems whose density is translationally invariant along $z$.

Solving Equation 27.4, or minimizing directly the functional of Equation 27.2, forms the basis for two different computational approaches that are discussed in the following sections.

\subsection{Axially Symmetric Solutions}

At first, we assume that the electron charge distribution is rotationally and translationally invariant in $\phi$ and $z$ degrees of freedom, respectively. This approximation is in the same spirit as the assumption of spherical symmetry used in the simulation of atomic systems. Moreover, as we shall demonstrate explicitly later, the cylindrical symmetry of the external potential is retained by the electron density at least for $r_{\mathrm{s}}$ in the $2 \leq r_{\mathrm{s}} \leq 6$ range appropriate for the valence charge of simple metals.

Cylindrical symmetry ensures the wave function is separable and may be written as

$$
\psi_{k_{z}}(\mathbf{r})=\chi(r) e^{i m \phi} e^{i\left((2 \pi / L) l+k_{z}\right) z}
$$

where $m$ and $l$ are relative integers, and, following widely accepted conventions, $k_{z}$ is selected in the interval $-(\pi / L) \leq$ $k_{z}<(\pi / L)$. All the states corresponding to a given set of quantum numbers $\{n m \sigma\}$ are referred to as a sub-band.

The radial function, $\chi(r)$, is then determined by solving the differential equation

$\frac{\mathrm{d}^{2} \chi(r)}{\mathrm{d} r^{2}}+\frac{1}{r} \frac{\mathrm{d} \chi(r)}{\mathrm{d} r}+\left[2\left(\varepsilon-V_{\mathrm{KS}}(\mathbf{r})\right)-\left(\frac{2 \pi l}{L}+k_{z}\right)^{2}-\frac{m^{2}}{r^{2}}\right] \chi(r)=0$

subject to the appropriate boundary conditions, that for bound states read

$$
\begin{gathered}
\lim _{r \rightarrow 0} \chi(r)=r^{m}\left(a_{0}+a_{1} r+a_{2} r^{2}\right) \\
\lim _{r \rightarrow \infty} \chi(r)=\frac{\exp (-\sqrt{-\varepsilon r})}{\sqrt{r}}
\end{gathered}
$$

These conditions can be satisfied for a discrete set of negative eigenvalues, that we indicate with $\varepsilon_{n m}\left(k_{z}+(2 \pi l / L)\right)$, where $n$ is a positive integer analogous to the principal quantum number of atoms. The corresponding radial functions, $\chi(r)$, depend on the $n, m$ quantum numbers, while they are independent of $l, k_{z}$, and in what follows they will be denoted by $\chi_{n m}(r)$. In addition to these bound states, the system has a continuum of scattering states whose eigenvalues are positive. The ground state density is given by

$$
\rho(r)=\sum_{n m l} \int_{\mathrm{BZ}} f\left[\varepsilon_{n m}\left(k_{z}+\frac{2 \pi l}{L}\right)\right]\left|\chi_{n m}(r)\right|^{2} \mathrm{~d} k_{z}
$$

where $f[\varepsilon]$ is the occupation number. In the case of a cylindrical ground state, the $z$-momentum dependence of $\varepsilon_{n m}\left(k_{z}\right)$ is given by

$$
\varepsilon_{n m}\left(k_{z}\right)=\varepsilon_{n m}\left(k_{z}=0\right)+\frac{k_{z}^{2}}{2}
$$

and the integration over $k_{z}$ can be performed analytically (Zabala et al. 1998). States are occupied up to an energy $\varepsilon_{F}$ such that the number of states whose energy is less than $\varepsilon_{F}$ is equal to the number of electrons in the system. The total number of distinct $\{n m\}$ combinations found for the occupied states is by definition the number of occupied sub-bands for the wire under investigation. As detailed below, this number is of the order of 10 for the sizes investigated in our study.

In our computation, a radial grid of 2000 points has been used, extending up to $R_{\max }=4 R_{\mathrm{c}}$ in the case of high density samples $\left(r_{\mathrm{s}}<10\right)$, and up to $R_{\max }=2 R_{\mathrm{c}}$ for low-density systems. Each orbitals is integrated outward from $r=0$, and inward from $R_{\max }$ using a predictor-corrector method. The corresponding eigenvalue is determined by the matching of the two solutions at $R_{\mathrm{c}}$. Angular momenta up to $m=20$ have been considered, and degenerate levels are equally populated at all stages of the calculation. A similar computational procedure is adopted for the determination of the Coulomb potential from the charge density.

Apart from the $k_{z}$ label, and apart from obvious differences in the radial equations, the approach is completely analogous to methods routinely used to compute the electronic structure of atoms with the restriction to spherical symmetry, and high accuracy solutions can be obtained relatively easily with a limited computational effort. The method outlined above has been used several times to determine the ground state properties of jellium wires whose density has cylindrical symmetry (Stafford et al. 1997, Yannouleas and Landman 1997, Yannouleas et al. 1998). 


\subsubsection{Computations in the Radial-Cylindrical Approach}

The results obtained by the radial code under the restriction of cylindrical symmetry are summarized in Figure 27.8. Our data agree with those of previous computations (Zabala et al. 1998, Stafford et al. 1997) whenever a comparison is possible.

As pointed out in Zabala et al. (1998), the enhancement of the exchange interaction due to confinement, together with the degeneracies of cylindrical wave functions in very thin wires, may gives rise to a progressive filling of sub-bands reminiscent of Hund's rule in atoms. In turn, this implies that partial polarization may arise at densities as high as those of bulk sodium $\left(r_{\mathrm{s}}=4\right)$. The integral of the spin polarization density, $\rho_{\mathrm{s}}(\mathbf{r})=$ $\rho_{+}(\mathbf{r})-\rho_{-}(\mathbf{r})$, is small, amounting to a few electrons at most, and, therefore, the relative spin polarization is fairly low as soon as the wire radius exceeds monoatomic thickness. Moreover, as expected, $\rho_{s}(\mathbf{r})=\rho_{+}(\mathbf{r})-\rho_{-}(\mathbf{r})$ always peaks at the background edge in high density samples, and spin polarization might indeed represent the precursor of spin-polarized states quasilocalized at the surface of the semi-infinite jellium.

Extensive spin polarization in the ground state appears only at much lower density, far below those found in elemental metals, but achievable in artificial conductors obtained by doping semiconducting structures or polymeric chains. A plot of the spin polarization energy $\Delta E_{\mathrm{S}}(\zeta)=E_{\text {tot }}(\zeta)-E_{\text {tot }}(0)$ for the wire and for the homogeneous electron gas shows that at low density and for the relatively thick wires considered in our study the stabilization of the spin polarization brought about by confinement and by inhomogeneity is fairly modest (see Figure 27.9). Nevertheless, computations in the cylindrical symmetry approximation show that the transition to a (partially) spin-polarized ground state

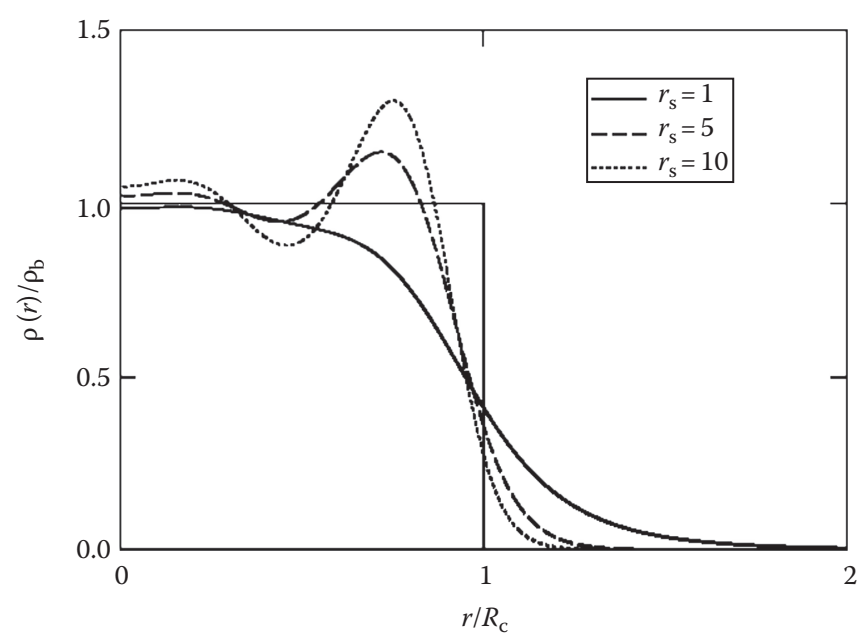

FIGURE 27.8 Electron density, $\rho(r)$, as a function of the radial coordinate, $r$, at three values of the electron gas parameter, $r_{\mathrm{s}}$, computed using the $1 \mathrm{D}$ algorithm under the assumption of cylindrical symmetry. The positive density distribution has radius $R_{\mathrm{c}}=3.162 r_{\mathrm{s}}$, and the ground state is paramagnetic for the three cases displayed here. (From Hughes, D. and Ballone, P., Phys. Rev. B, 77, 245312, 2008. With permission.)

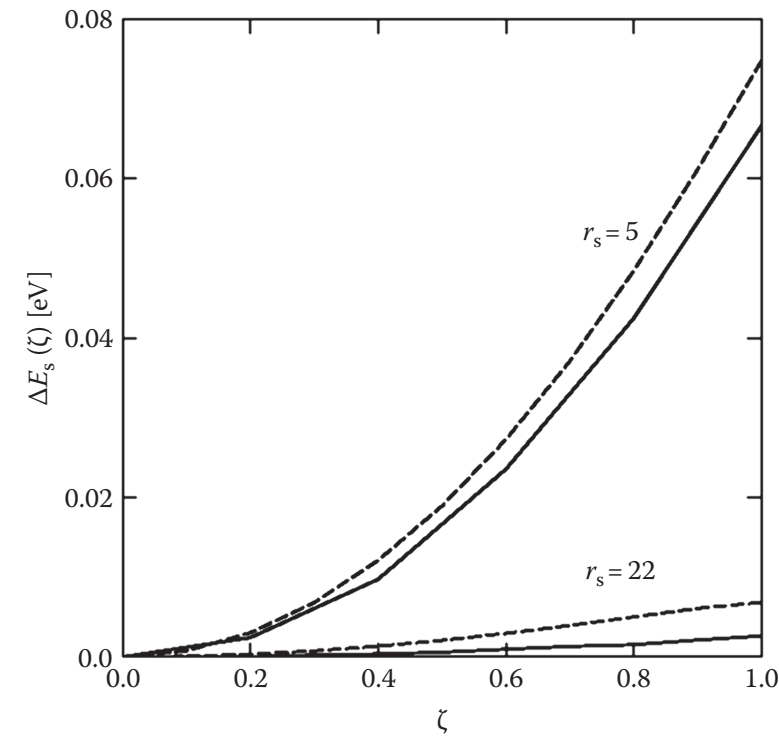

FIGURE 27.9 Spin-polarization energy $\Delta E_{\mathrm{s}}(\zeta)=E_{\text {tot }}(\zeta)-E_{\text {tot }}(0)$ for the wire (full line) and for the homogeneous electron gas (dash line). Computations have been performed for a finite system with $N=240$ electrons under the assumption of a cylindrically symmetric charge density. (From Hughes, D. and Ballone, P., Phys. Rev. B, 77, 245313, 2008. With permission.)

takes place at $r_{\mathrm{s}}=27$. At variance from what has been found in high-density wires, the spin polarization in low-density, low (nominal $\zeta \sim 0$ ) spin polarization samples tends to be localized in the central region of the wire, with only negligible contributions from regions beyond the background radius.

\subsection{Iterative Minimization of the LSD Density Functional}

The restriction to cylindrical symmetry for the electron density has to be abandoned in order to describe broken symmetry solutions such as the WC, expected to arise at low $\rho_{\mathrm{b}}$ densities, and also in the case of wires subject to an external perturbation not conserving the original symmetry. In those cases, a general solution may be obtained by expanding KS orbitals in plane waves:

$$
\psi_{\mathrm{k}}^{(i)}(\mathbf{r})=\sum_{\mathbf{G}} c_{\mathrm{G}}^{(i)} e^{i \mathbf{G r}} e^{i \mathbf{k r}}
$$

In doing so we assume that the system is periodic in $3 \mathrm{D}$, and we impose a fictitious periodicity of length $\left(L_{x}, L_{y}\right)$ in the plane perpendicular to the $z$ axis. Among other things, this periodicity implies that the label $\mathbf{k}$ is now a $3 \mathrm{D}$ vector. The plane wave expansion of Equation 27.11 is limited to those $\mathbf{G}$ vectors such that $|\mathbf{G}+\mathbf{k}|^{2}$ is less than a preselected cutoff $E_{\text {cut }}$ which has the dimensions of an energy, and in what follows is measured in Rydbergs.

The functional $E_{K S}\left[\left\{\psi_{i}\right\}\right]$ is in fact a function of the multitude of $\left\{c_{\mathbf{G}, \mathbf{k}}^{(i)}\right\}$ coefficients, and it can be optimized by iterative minimization, using the information provided by the gradient: 


$$
\begin{aligned}
\frac{\partial E_{\mathrm{KS}}}{\partial c_{\mathbf{G}, \mathbf{k}}^{(i)}} & =\sum_{\mathbf{k}} \int \frac{\delta E_{\mathrm{KS}}}{\delta \psi_{\mathrm{k}}^{(i)}(\mathbf{r})} \frac{\mathrm{d} \psi_{\mathrm{k}}^{(i)}(\mathbf{r})}{\mathrm{d} c_{\mathbf{G}}^{(i)}} \mathrm{d} \mathbf{r}=\sum_{\mathbf{k}} \int \frac{\delta E_{\mathrm{KS}}}{\delta \psi_{\mathrm{k}}^{(i)}(\mathbf{r})} e^{i \mathrm{Gr}} \mathrm{d} \mathbf{r} \\
& =\sum_{\mathbf{k}} \int\left\{-\frac{1}{2} \nabla^{2}+V_{\mathrm{Ha}}(\mathbf{r})+\mu_{\mathrm{XC}}[\rho]\right\} \psi_{\mathrm{k}}^{(i)}(\mathbf{r}) e^{i \mathrm{Gr}} \mathrm{d} \mathbf{r}
\end{aligned}
$$

The sum over $\mathbf{k}$ points is a discretized version of the integral over the $\mathrm{BZ}$ implied by the $3 \mathrm{D}$ periodicity.

The actual optimization may be achieved by a variety of methods (Marx and Hutter 2000), including steepest descent, conjugate gradient, etc. In all cases, the energy minimization is performed at fixed spin polarization $\zeta=\left(n_{+}-n_{-}\right) /\left(n_{+}+n_{-}\right)$, where $n_{+}$and $n_{-}$are the spin-up and spin-down charges, respectively. The sum of $n_{+}$and $n_{-}$is equal to $N$, although neither of the two partial charges needs to be an integer if the system is a metal.

As described below, for low $r_{s}$ (high density) and up to at least $r_{\mathrm{s}}=20$ the ground state density turns out to be cylindrically symmetric, and the cutoff energy for the 3D-plane wave computation is tuned by the comparison with the results of the radial program using full cylindrical symmetry. Total energies computed by the two methods shows that convergence in the plane wave expansion is already achieved at a cutoff energy of $E_{\text {cut }}\left(r_{\mathrm{s}}\right) \sim 10 / r_{\mathrm{s}}^{2}$ Ry. The localized states found at low density $\left(r_{\mathrm{s}} \geq 30\right)$ are more difficult to represent in plane waves, and explicit tests have shown that a cutoff energy of $E_{\text {cut }}=20 / r_{\mathrm{s}}^{2} \mathrm{Ry}$ is required for a uniform convergence of ground state properties over the full density range $1 \leq r_{s} \leq 100$ explored in our study. Despite the relatively low cutoff, the large cell size implies that the number of variational degrees of freedom is of the order of $5 \times 10^{4}$ per state. Taking into account the high number of states included in our computations, it is not surprising to find that the minimization of the energy functional turns out to be relatively time consuming.

We emphasize that while the nominal (or net) spin polarization, $\zeta=\left(N_{+}-N_{-}\right) /\left(N_{+}+N_{-}\right)$, is an input parameter of our computations, the spin density, $\rho_{s}(\mathbf{r})=\rho_{+}(\mathbf{r})-\rho_{-}(\mathbf{r})$, is fully unconstrained both in the radial cylindrical and in the plane wave approach, and $I_{\mathrm{s}}=\int\left|\rho_{\mathrm{s}}(\mathbf{r})\right| \mathrm{d} \mathbf{r}$ can be significantly larger than $\left|\mathrm{N}_{+}-\mathrm{N}_{-}\right|$. In particular, at low background density $\left(r_{\mathrm{s}}>30\right)$ local spin polarization is found also in nominally paramagnetic samples at $\zeta=0$.

\subsection{Broken-Symmetry Solutions}

Computations have been performed for a series of wires of length $L=32 r_{\text {s }}$ periodically repeated along $z$, consisting of $N=240$ electrons moving in the electrostatic potential of a cylindrical distribution of positive charge whose plasma parameter $r_{\mathrm{s}}$ spans the range $1 \leq r_{\mathrm{s}} \leq 100$. At $r_{\mathrm{s}}=1$, therefore, the radius of the positive charge is $R_{\mathrm{c}}=3.162$ a.u., or $1.673 \AA$, and the wire segment explicitly included in the computation is $16.93 \AA$ long. The radius reaches $167.3 \AA$ at the lower density range $\left(r_{\mathrm{s}}=100\right)$, and in such a case the periodicity along $z$ is $1693 \AA$ or $0.1693 \mu \mathrm{m}$. All wires have the same aspect ratio, $L / R_{\mathrm{c}}=10.12$.
Plane wave computations have been performed using a cubic simulation box of side $L$, and, therefore, the background density occupies $\sim 3 \%$ of the simulation box.

Because of the fairly large size of our samples, and considering also their low average density, the sampling of the system $\mathrm{BZ}$ in the plane wave computations has been restricted to the $\Gamma$-point only. The role of $k_{z}$ points is decreased by the fact that the systems we are primarily interested in (i.e., the low-electrondensity wires) are in fact insulators.

The full range of spin polarizations, $0 \leq \zeta \leq 1$, has been explored by varying the relative number of spin-up $\left(N_{+}\right)$and spin-down $\left(N_{-}\right)$electrons, starting from the paramagnetic case $\left(N_{+}=N_{-}=120\right)$, and progressively increasing (decreasing) $N_{+}\left(N_{-}\right)$in steps of 10 electrons up to $N_{+}=240\left(N_{-}=0\right)$. We use here $N_{+}$and $N_{-}$instead of $n_{+}$and $n_{-}$to indicate that the net spin imbalance, $N_{\text {spin }}=N_{+}-N_{-}$, is restricted to integer values.

\subsubsection{Plane Wave Computations}

Electron localization is fully accounted for by the 3D-plane wave computations, whose results display the same magnetic transition at nearly the same density of the radial-cylindrical computations. More precisely, up to $r_{\mathrm{s}}=30$ the solutions found by the plane wave code also display translational invariance along $z$ at all $\zeta$, and, apart from occasional interchanges of nearly degenerate states, the corresponding sequence of KS orbitals agrees with that of the radial computations for full cylindrical symmetry. As anticipated in Section 27.5, the convergence of the plane wave expansion is confirmed by the good agreement of the total energy obtained by the two computational approaches.

Sizable differences between the solutions of the radial scheme and those of the plane wave computation first appear at $r_{\mathrm{s}}=30$. Inspection of the electron density found by the unconstrained plane wave minimization (see Figure 27.10) reveals that an apparent localization transition involving all coordinates (i.e., now including $z$ ) takes place in the samples of highest spin polarization $(\zeta \geq 0.5)$. Localization can be described as partial because the overlap of different electron-density peaks is significant, and the density at local minima is still a sizable fraction of $\rho_{b}$ (see the inset in Figure 27.10). We verified that the density modulation along $z$ remains nearly unchanged when the sampling of the BZ is extended to more $k_{z}$ points. Further analysis described below suggests that the electron configuration consists of a majority of delocalized states similar to those found at high density, coexisting with localized states whose energy is at the bottom and at the top of the occupied (valence) band.

We remark that up to $r_{\mathrm{s}}=40$ localization in the radial direction, giving rise to a sequence of well-defined electron-density shells, is more marked than localization within each of the radial shells. Moreover, localization is stronger in the inner region of the wire, and somewhat attenuated in the outer electron shell, as apparent in Figure 27.10.

Comparison of the different energy contributions for the z-invariant and for the localized states show that, as expected, localization is driven by a gain in correlation energy, only partly 


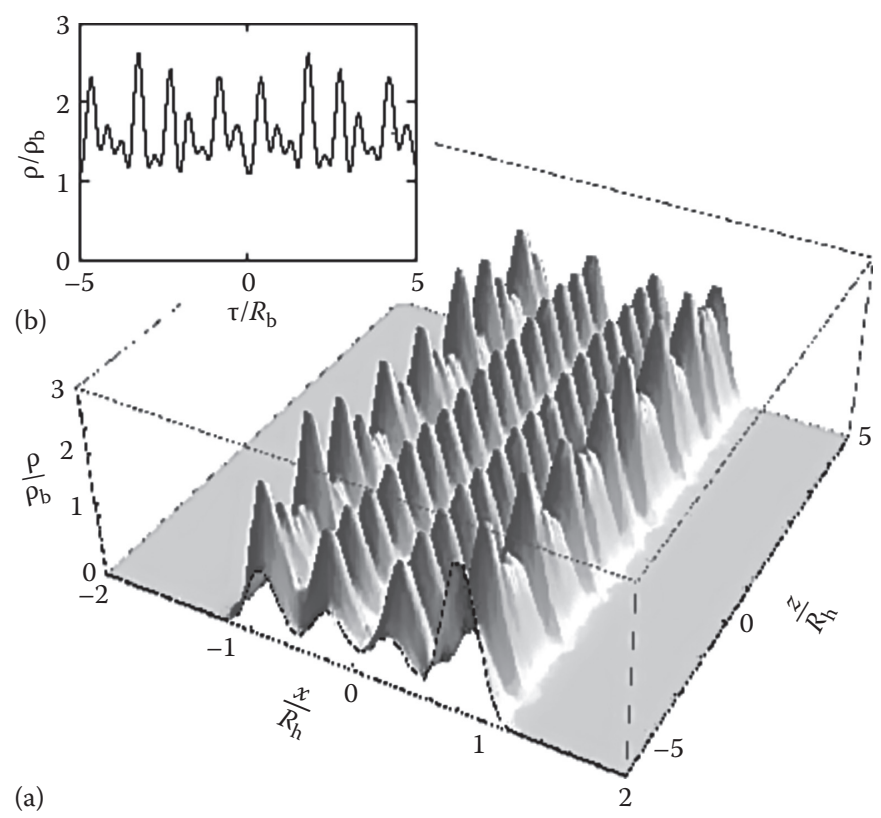

FIGURE 27.10 Electron density of a fully spin-polarized jellium wire obtained by the plane wave method. $N=240, r_{\mathrm{s}}=30$. Panel (a): $2 \mathrm{D}$ plot of the density $\rho(r, z)$ on the axial plane $\phi=0$. Panel (b): 1D plot of the electron density, $\rho(z)$, along the line parallel to the cylindrical axis of coordinates: $x-a=y=0$, where $a=2.25 \times r_{\mathrm{s}}=0.71 R_{\mathrm{c}}$. (From Hughes, D. and Ballone, P., Phys. Rev. B, 77, 245314, 2008. With permission.)

compensated by the kinetic energy term, which increases upon localization. This energy balance, in turn, explains why localization takes place at first in spin-polarized samples, since the kinetic energy (Ashcroft and Mermin 1976) of ferromagnetic states is higher than that of the paramagnetic state, while their correlation energy is lower. On the other hand, the kinetic energy of the WC is nearly independent of spin, and thus, the kinetic energy cost of localized states is less relevant for the ferromagnetic configuration, while the potential gain in correlation energy is comparatively larger. Both energy terms, therefore, point to high spin configurations as the first candidates for localization.

Despite the energy gain provided by localization, at $r_{\mathrm{s}}=30$ the ferromagnetic configuration is still slightly higher in energy than the paramagnetic, $z$-invariant state. The energy difference between the two, however, is very small, and, in fact, the ground state energy is almost constant over the entire $0 \leq \zeta \leq 1$ range, suggesting that in the vicinity of the localization transition spin glass features might arise from the near degeneracy of several different spin states. This same near degeneracy with respect to changes of $\zeta$ makes it difficult to provide an accurate determination of the net ground state spin polarization at densities close to the transition point (see the inset in Figure 27.11). Nevertheless, plane wave computations confirm the stability of partially polarized stated at $r_{\mathrm{s}}$ slightly higher than 30 , as already anticipated by the radial-cylindrical computations. Of course, these observations imply that the transition to the spin-polarized state is at most weakly first order.

The fully ferromagnetic state becomes the state of lowest energy for $r_{\mathrm{s}} \geq 35$, the shift in the transition point from $r_{\mathrm{s}}=27$ estimated in the radial-cylindrical method being due to the discretization

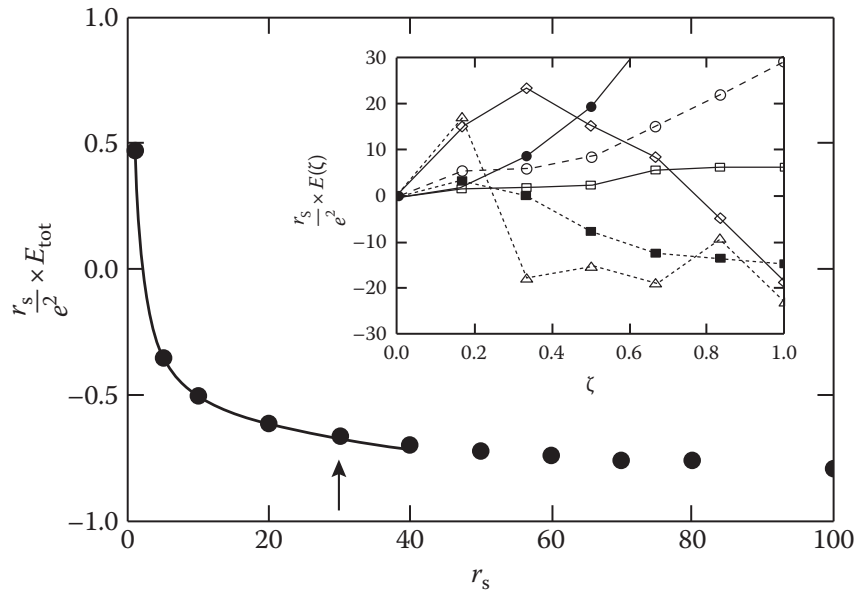

FIGURE 27.11 Total energy per electron of jellium wires computed by the radial-cylindrical method (full line) and by the 3D-plane wave method (full dots). At each $r_{s}$, the energy of the lowest energy spin configuration is reported. The arrow marks the transition between paramagnetic and partially spin-polarized configurations. The polarization energy per electron, $\Delta E_{\mathrm{s}}(\zeta)$, at six background densities is shown in the inset. Full dots: $r_{\mathrm{s}}=1$; circles: $r_{\mathrm{s}}=20$; squares: $r_{\mathrm{s}}=30$; filled squares: $r_{\mathrm{s}}=40$; diamonds: $r_{\mathrm{s}}=70$; triangles: $r_{\mathrm{s}}=100$. (From Hughes, D. and Ballone, P., Phys. Rev. B, 77, 245315, 2008. With permission.)

of the DOS, and to the finite plane wave expansion of orbitals and electron density, which affects spin-polarized systems slightly more than the paramagnetic ones. In the low-density regime at $r_{\mathrm{s}} \geq 40$, localization is apparent in all systems, irrespective of spin polarization, and becomes progressively more marked with increasing $r_{s}$. Plots of constant electron-density surfaces (isosurfaces, in what follows) provide a direct and intuitive view of 


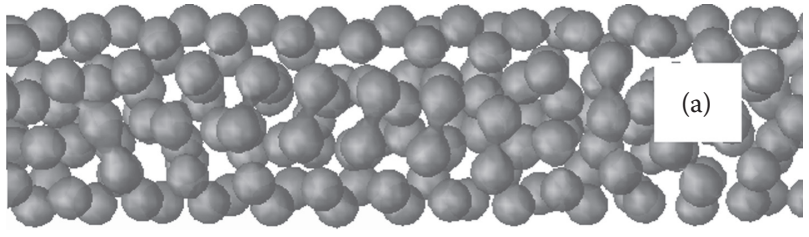

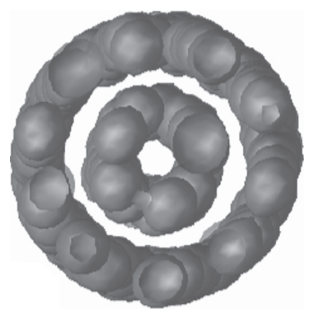

(b)

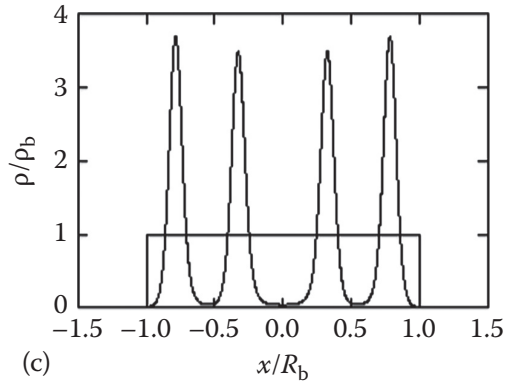

FIGURE 27.12 Electron-density contour plot for the ferromagnetic ground state of the $r_{\mathrm{s}}=70, N=240$ wire. Panel (a): side view. Panel (b): transversal view. Panel (c): radial density profile obtained upon averaging the $3 \mathrm{D}$ density over $\phi$ and $z$. (From Hughes, D. and Ballone, P., Phys. Rev. B, 77, 245316, 2008. With permission.)

the localization extent in low-density systems, as apparent from Figure 27.12) showing the $\rho=1.6 \rho_{\mathrm{b}}$ isosurface for the ferromagnetic ground state at $r_{\mathrm{s}}=70$. Shell effects are apparent from the transversal view of the density distribution (Figure 27.12b and c), and might be seen as the oversized and frozen-in version of the charge (Friedel) oscillations already present in the high density liquid phase (see Figure 27.8). The perspective view of the same isosurface (Figure 27.12a) clearly shows that the system consists of an assembly of well-defined charge droplets. In what follows, these droplets will sometimes be referred to as charge blobs, to account for their somewhat irregular shape.

The gradual organization of charge into shells with increasing $r_{s}$ followed by the breakdown of shells into one-electron droplets is qualitatively similar to the two-stage freezing (or, equivalently, melting) observed in 2D circular quantum dots (Filinov et al. 2001), whose radial and orientational order are set in at different densities and/or temperatures. Only for sufficiently large systems, the two localization processes merge into a unique freezing transition.

The fairly regular pattern displayed by the droplets distribution in low-density samples suggests that a geometrical lattice, possibly closely related to the bcc structure of the extended WC, might underlay the ground state charge configuration. To identify this ideal geometry, the continuous density distribution provided by LSD is mapped onto a particles configuration by (1) first identifying connected regions whose density is higher than $\rho_{\text {cut }}=2 \rho_{b}$, and then (2) associating one particle to each of these domains, and locating it at the center of mass of the corresponding charge distribution. This procedure provides a fully unambiguous result only for systems of fairly high $r_{s}\left(r_{s}>50\right)$. For these low-density systems, the number of connected regions (and thus the number of associated particles) is always very close to the number $N$ of electrons in the system, the difference being at most a few units in all samples at $r_{\mathrm{s}} \geq 70$, thus lending a reality flavor to the representation of the electron density by particles. The configuration obtained in this way closely resembles the low-temperature structure of classical particle models such as the one-component plasma (OCP), as obtained by slowly annealing liquid samples. In this respect, it is interesting to note that the radial distribution function, $g(r)$, of the representing particles belonging to the inner radial shells of the computed structures displays the same characteristic features found in the glassy state of the classical OCP (Tanaka and Ichimaru 1987), consisting in an asymmetric first peak and a split-second peak (see Figure 27.13).

The radial distribution function of particles representing charge blobs depends only weakly on spin polarization (see below) and on density for $r_{\mathrm{s}} \geq 70$, apart from a trivial scaling of all distances. The dependence on sample size is also very weak up to the second peak of $g(r)$, while it becomes important at larger distances. These results are reflected in the weak density and size dependence of the running coordination number, $n_{\mathrm{c}}(r)$, defined as

$$
n_{\mathrm{c}}(r)=4 \pi \rho_{\mathrm{b}} \int_{0}^{r} r^{\prime 2} g\left(r^{\prime}\right) \mathrm{d} r^{\prime}
$$

and displayed in the inset of Figure 27.13.

Despite the unambiguous mapping of charge blobs into particles, the identification of the ideal structure underlying the ground state charge distribution of low-density wires is made

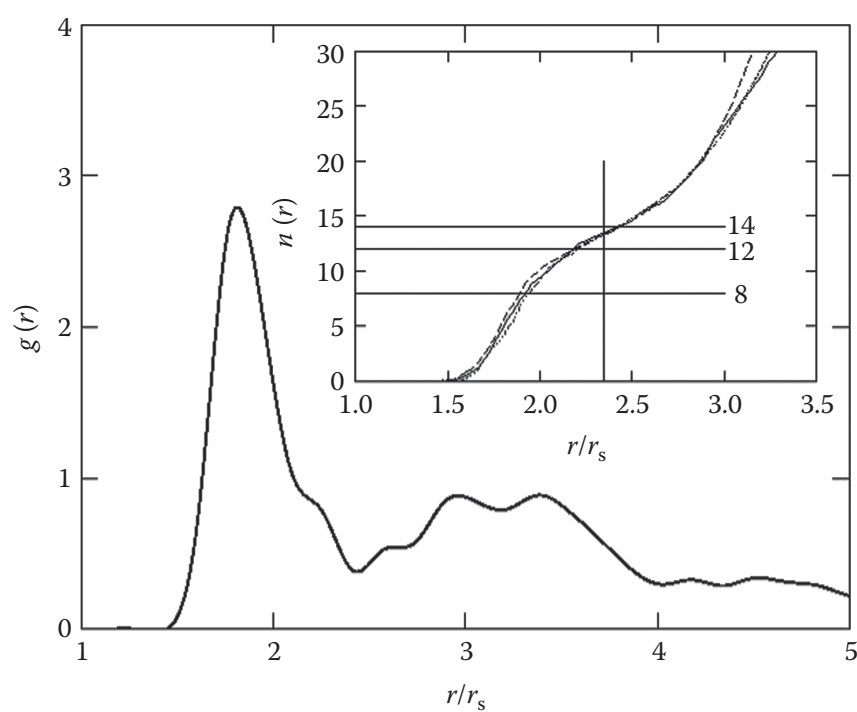

FIGURE 27.13 Radial distribution function of particles representing charge blobs (see text) for a wire of $N=240$ electrons at $r_{\mathrm{s}}=70$ and $\zeta=1$. Inset: running coordination number, $n_{\mathrm{c}}(r)$, of particles representing charge blobs. Full line: $N=240$ electrons, $r_{\mathrm{s}}=70, \zeta=1$; dash line: $N=480$ electrons, $r_{\mathrm{s}}=70, \zeta=1$; dotted line: $N=240$ electrons, $r_{\mathrm{s}}=100$, $\zeta=1$. The horizontal lines correspond to full shells of neighbors in the bcc $\left(n_{\mathrm{c}}=8\right.$ and $\left.n_{\mathrm{c}}=14\right)$ and in the fcc $\left(n_{\mathrm{c}}=12\right)$ lattice. The vertical line corresponds to the minimum of $g(r)$, and defines the cutoff radius for the computation of the average coordination number. (From Hughes, D. and Ballone, P., Phys. Rev. B, 77, 245317, 2008. With permission.) 


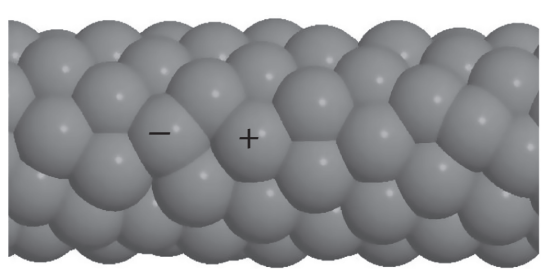

(a)

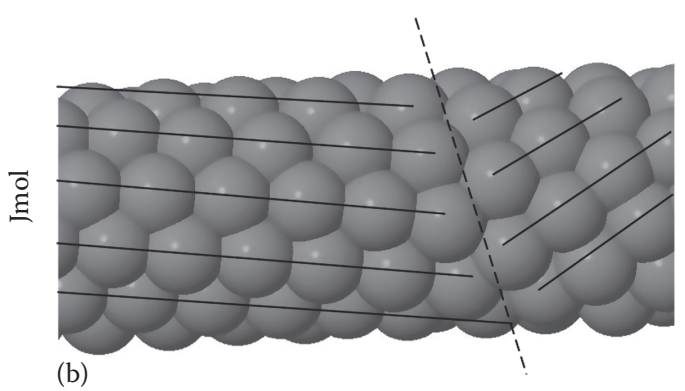

FIGURE 27.14 Defective configurations in the ground state distribution of charge droplets of fully spin-polarized wires at $r_{\mathrm{s}}=70$ : (a) pair $(+,-)$ of miscoordinated droplets in the middle shell of the $N=480$ sample; (b) grain boundary on the inner shell of the $N=240$ sample. (From Hughes, D. and Ballone, P., Phys. Rev. B, 77, 245318, 2008. With permission.)

difficult by the unavoidable distortions imposed by the finite sample size, and by the likely mismatch of the optimal lattice parameter with the other length scales entering the definition of our model, such as the background radius and the wire length. However, the major difficulty in characterizing the particles' geometry arises from a variety of point and extended defects such as dislocations and grain boundaries that are distributed in the structures produced by our energy optimization (see Figure 27.14) both for the $N=480$ (Figure 27.14a) and for the $N=240$ systems (Figure 27.14b). These defects are likely to result, at least to some extent, from limitations of our computational scheme, unable to reach the absolute minimum of the DF energy within an acceptable number of iterations. More importantly, the disorder in the electron droplets distribution certainly reflect a real and relevant property of these low energy systems, having a vast number of similar but different configurations of nearly equal energy. In turn, the positional disorder frozen into low energy configurations is likely to affect the properties of electrondensity systems measured in experiments, and to give rise to glass-like features in the thermodynamics and real-time dynamics of low-density wires.

Despite the unavoidable uncertainties due to the intrinsic disorder of the structures resulting from our computations, information on the underlying ground state structure can be obtained from average quantities such as the radial distribution function, $g(r)$, and the coordination number, $n_{c}$, defined as the value of the running coordination number up to a distance corresponding to the first minimum of the radial distribution function $\left(r_{\min }=2.3 r_{\mathrm{s}}\right.$ for wires of $\left.r_{\mathrm{s}} \geq 50\right)$. The similarity of the particles' $g(r)$ with those of the OCP already pointed out above clearly suggests a close relation with a bcc lattice. The coordination number, $n_{c}$, of the charge blobs residing in the inner shell of the computed structures is close to but nevertheless systematically lower than the $n_{\mathrm{c}}=14$ value that corresponds to the number of first and second neighbors in the bcc structure (see Figure 27.13). However, the absence of the shell closing at $n_{\mathrm{c}}=8$ also expected for bcc, once again prevents a fully unambiguous identification. A detailed analysis of the structures found for the $N=240$ and $N=480$ samples however suggest that it might be more appropriate to characterize the computed geometries as being intermediate between an fcc and a bcc lattice.
At the highest $r_{\mathrm{s}}$ 's considered in our study $\left(r_{\mathrm{s}} \geq 70\right)$, the separation of the density peaks is so marked that it is possible and even easy to identify the spatial domain occupied by each blob. This allows us to verify that not only the number of droplets corresponds to the number $N$ of electrons, but, in addition, the integral of the charge density for each blob is very close to one, the standard deviation amounting to only $5 \%$. At low density, therefore, blobs can be identified with electrons, even though they should not be identified with KS orbitals, as it will be discussed later. The remarkable correspondence of density blobs and electrons arises from well-known anomalies in the response functions, that in reciprocal space identify the BZ of the WC, and in real space delimit the lattice unit cell, thus determining the size, charge, and spin of the basic building block.

Individual charge blobs always display partial (at $r_{\mathrm{s}}<70$ ) or full $\left(r_{s} \geq 70\right)$ spin polarization for all systems in which localization is apparent, irrespective of the average polarization $\zeta$ and including nominally paramagnetic samples. Needless to say, this implies that the electronic structure of low- $\zeta$ systems includes a spin-compensating mechanism, such as antiferromagnetic ordering or a more general spin wave, bringing the net spin to the value imposed by the $N_{+}$and $N_{-}$values. This is apparent in Figure 27.15, displaying the spin polarization of charge blobs for the wire $r_{\mathrm{s}}=70, \zeta=0$ wire. The spin configuration of the outermost shell is fairly disordered, while the inner shell displays a regular helical pattern (not very clear in Figure 27.15, as in any $2 \mathrm{D}$ representation, but apparent in computer visualizations

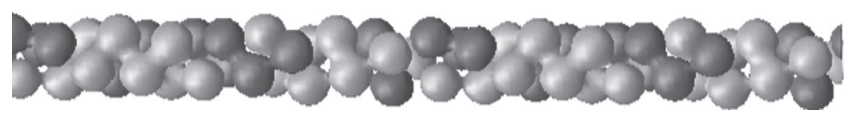

(a)

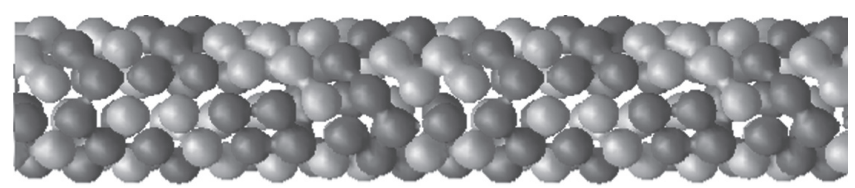

(b)

FIGURE 27.15 Spin polarization of charge droplets for the $r_{\mathrm{s}}=70$, $\zeta=0$ wire. Red: spin-up droplets. Blue: spin-down droplets. The inner (a) and outer (b) shells are shown separately. (From Hughes, D. and Ballone, P., Phys. Rev. B, 77, 245319, 2008. With permission.) 
that allow one to rotate the isosurface) probably related to the enhancement of the spin-spin response function at $k_{z}=2 K_{F}$ and $k_{z}=4 K_{F}$ (Pouget and Ravy 1997).

The disordered spin distribution of the outer shell suggests that the spin-spin coupling constant is fairly small, as confirmed by the computation of the spin-resolved radial distribution functions $g_{++}(r)$ and $g_{+-}(r)$ that show only a slight predominance of antiferromagnetic coupling in the first coordination shell. This could be seen as the expected consequence of a nearly disjoint charge and spin blobs, reducing also the exchange interaction. It is important to realize, however, that localization concerns the density, not necessarily the KS orbitals. We verified, in fact, that even in samples displaying the most apparent charge localization, each KS orbital contributes to the density of several, widely spaced blobs, as can be seen in Figure 27.16. The qualitative information contained in this figure is confirmed by a quantitative measure of localization provided by the computation of the inverse participation ratio, that, apart from a few cases, points to a remarkably low localization for KS orbitals, as discussed below.

Charge and spin localization are nevertheless clearly reflected into basic properties of the KS orbitals, affecting, for instance, the system DOS. As can be seen in Figure 27.17, the density of states for the $r_{s}=30, \zeta=1$ sample shows a deep minimum (pseudogap) at the Fermi energy, pointing to an incipient metal-insulator
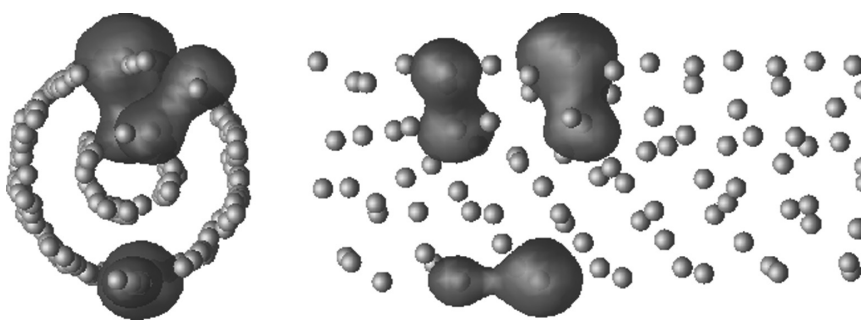

FIGURE 27.16 Density isosurface, $\rho_{1}=0.05 \rho_{\mathrm{b}}$, for a KS orbital whose eigenvalue is close to the top of the occupied band. $r_{\mathrm{s}}=70, N=240$, fully spin-polarized ground state. Solid particles mark the center-of-mass position of individual charge blobs. (From Hughes, D. and Ballone, P., Phys. Rev. B, 77, 245320, 2008. With permission.) transition driven by localization. The $r_{\mathrm{s}}=40, \zeta=1$ sample is clearly an insulator, and at $r_{\mathrm{s}}=70, \zeta=1$ the energy gap separating occupied and unoccupied states is as wide as the total width of the occupied bands.

The localization of individual orbitals is measured by computing the inverse participation ratio, defined as follows. First of all, we compute the $z$-dependent planar average of each orbital, defined as

$$
\psi_{i}^{\text {plane }}(z)=\frac{\int \psi_{i}(x, y, z) \mathrm{d} x \mathrm{~d} y}{\pi R_{\mathrm{c}}^{2}}
$$

then, the inverse participation ratio, $p_{i}^{-1}$, is computed according to

$$
p_{i}^{-1}=L \frac{\int\left|\psi_{i}^{\text {plane }}(z)\right|^{4} \mathrm{~d} z}{\left[\int\left|\psi_{i}^{\text {plane }}(z)\right|^{2} \mathrm{~d} z\right]^{2}}
$$

The definition implies that localized states correspond to $p^{-1} \sim L$, and delocalized states to $p^{-1} \sim 1$. The results, reported in Figure 27.17, show that the central and major portion of the occupied band is made of fairly delocalized states, while the most localized orbitals are found at the low- and high-energy band edges. While the localization of these states could have been expected, the relative delocalization of all the other states is more surprising, given the apparent strong localization of the charge.

The different behavior of the density and of KS orbitals with respect to localization might be related to the invariance of LSD with respect to unitary transformation of the occupied states, that, by definition, leave the electron density and the kinetic energy unchanged. All the effects described in this section are likely to affect the transport properties of nearly $1 \mathrm{D}$, low-carrier density conductors.

As a last observation, we would like to mention that at the lowest densities explored by our computations $\left(80 \leq r_{\mathrm{s}} \leq 100\right)$, the plane wave energy optimization gives rise to surprising new structures, especially for the low-spin samples, as shown in Figure 27.18 for the $N=240$ wire of $r_{\mathrm{s}}=100, \zeta=1$.

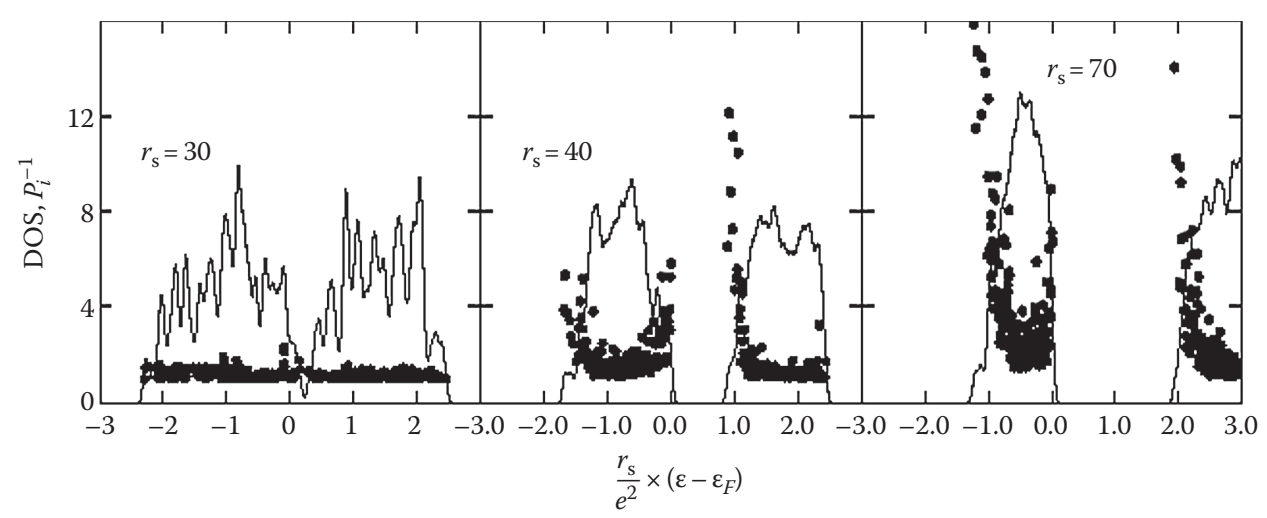

FIGURE 27.17 Density of states and inverse participation ratio, $p_{i}^{-1}$, for the fully spin-polarized wires at $r_{\mathrm{s}}=30,40$, and $70, N=240$. (From Hughes, D. and Ballone, P., Phys. Rev. B, 77, 245321, 2008. With permission.) 


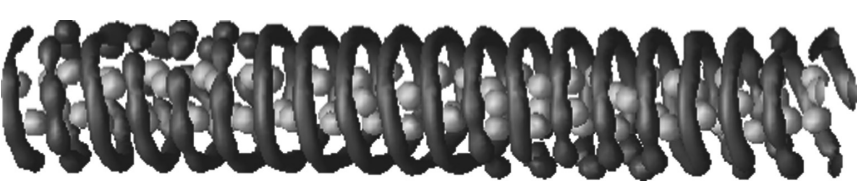

FIGURE 27.18 Density isosurface, $\rho=2 \rho_{\mathrm{b}}$, for the sample of $N=240$ electrons, $r_{\mathrm{s}}=100, \zeta=0$. (From Hughes, D. and Ballone, P., Phys. Rev. $B$, $77,245322,2008$. With permission.)

\subsection{Nano-Constriction: Preliminary DF Results}

The experiments of Bird et al. (Bird and Ochiai 2004, Yoon et al. 2007) point toward exotic magnetic behavior in QPC. By introducing a neck, or narrowing into the jellium background, we have been able to model such systems (Cortes-Huerto et al. 2009).

Once again, the background density is a constant within the wire and zero outside. The form of the neck is as follows:

$$
\rho_{N}(\mathbf{r})= \begin{cases}\rho_{\mathrm{b}} & \text { if } \gamma(z) \leq r \leq R_{b} \text { and }\left|z-z_{0}\right| \leq D \\ 0 & \text { otherwise }\end{cases}
$$

where

$\gamma(z)=r_{0}+\alpha\left(z-z_{0}\right)^{2}$ is the quadratic form of the constriction

A plot of the corresponding background density is shown in Figure 27.19.

The corresponding Fourier transform is

$$
\rho_{N}(\mathbf{G})=\frac{2 \pi \rho_{\mathrm{b}}}{V} \int_{z_{0}-D}^{z_{0}+D} \mathrm{~d} z \cos \left(G_{z} z\right) I\left(G_{r}, z\right)
$$

where

$$
I\left(G_{r}, z\right)= \begin{cases}\frac{1}{2}\left[R_{b}^{2}-\gamma(z)^{2}\right] & \text { if } G_{r}=0 \\ \frac{1}{G_{r}}\left[R_{b} J_{1}\left(G_{r} R_{b}\right)-\gamma(z) J_{1}\left(G_{r} \gamma(z)\right)\right] & \text { otherwise }\end{cases}
$$

and $G_{r}^{2}=G_{x}^{2}+G_{y}^{2}$.

We shall not describe in detail the results of these computations that are still partially preliminary. However, we include a plot of the magnetization isosurfaces, where the positiondependent magnetization is defined by $m(\mathbf{r})=n_{\uparrow}(\mathbf{r})-n_{\downarrow}(\mathbf{r})$, in an $r_{\mathrm{s}}=20$ system (Figure 27.20). Our findings reveal the

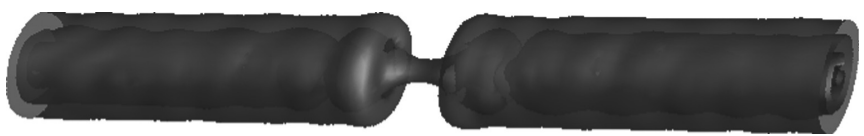

FIGURE 27.19 Electron density for a wire with a narrow geometric constriction.

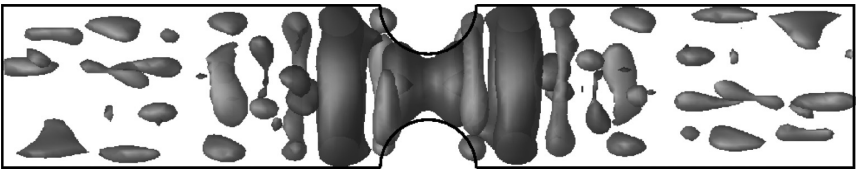

FIGURE 27.20 Magnetization density close to a nanoconstriction, $m(\mathbf{r})=0.20 \rho_{\mathrm{b}}$ (blue), $m(\mathbf{r})=-0.20 \rho_{\mathrm{b}}$ (red), background density $r_{\mathrm{s}}=25$, nominally paramagnetic sample of $N=230$ electrons.

formation of complex magnetic structures, such as rings close to the constriction. Work is in progress to assess the effect of these modulations of the spin density on properties such as DC and $\mathrm{AC}$ conductivity.

\subsection{Discussion and Conclusive Remarks}

Experimental and technological advances have raised great expectations on the potential applications of systems of nanometric size and on nanostructured materials. Among the systems of interest in this domain, nanowires represent a particularly important case, since every functional device will require the electrical connectivity of a vast amount of more elementary and equally nanometric components. Moreover, at the level of complexity achievable at this length scale, a network of conducting wires may acquire new functionalities (Yanushkevich and Steinbach 2008), going far beyond the passive role of traditional circuits.

Besides being important from the technological point of view, the conductivity of nanowires contains many features of interests from a more fundamental perspective. First of all, experimental measures for thin wires show that conductivity is quantized at values $2 e^{2} / \hbar$ (van Wees et al. 1988). This behavior is explained by standard scattering theory (Landauer 1970), but violations to this rule have been observed (Bird and Ochiai 2004, Yoon et al. 2007), pointing to spontaneously spin-polarized electronic configurations in otherwise fully paramagnetic systems (Thomas et al. 1996). More generally, the Tomonaga-Luttinger (Tomonaga 1950, Luttinger 1963) theory predicts that the normal FL theory familiar from 3D metals (Giuliani and Vignale 2005) does not apply to conductors all the way down to atomically thin wires, and has to be replaced by a different picture in which the dynamics of charge and spin degrees of freedom becomes progressively more decoupled (Fiete 2007), opening the way to a wealth of new phenomena. Finally, and perhaps more importantly for this chapter, a metal-nonmetal transition is predicted to take place with decreasing density of the charge carriers, due to the localization of electrons and/or holes under the effect of their mutual Coulomb interactions (Schulz 1993). This transition, conceptually similar to the well-known Wigner transition predicted long ago for the 3D homogeneous electron gas (Wigner 1934), is comparably more relevant in the case of Q1D conductors, since confinement and low dimensionality enhance the role of electron correlation, and amplify the effect of localization, which can completely block conduction even when it takes place in a finite portion of the entire system. 
In this chapter, we have provided a brief description of the phases believed to occur in 1D and Q1D systems. These include the "normal" regime, believed to be LL, which is contrasted to the regular FL; a magnetically ordered phase; a low-density, crystalline regime; and finally, other even more exotic phases identified by dynamical or excited-state properties. Moreover, we have summarized a number of theoretical studies, ranging from the use of simple idealized models to state-of-the-art correlated methods.

A short summary of the experimental methods that have been used to fabricate wires of nanometric diameter over a wide carrier density range has been provided in Section 27.3, The summary aims at emphasizing that the low-density nanometric wires that are the subject of this chapter are not just theoretical or computational abstractions.

In the second half of this chapter, we introduced an idealized (jellium) model of metal wire, and we investigated the localization transition by a conceptually simple and computationally tractable approach based on the DF theory (Hohenberg and

AQ5 Kohn 1964, Kohn and Sham 1965). The approximation we use (LSD) to treat exchange and correlation has been specifically devised for electron systems in a low correlation regime, and the quantitative predictions of the method for the highly correlated states found in low-density nanowires has to be taken with some caution. However, the method and the results it provides have a great didactical value, since they describe the system and its localization transition in simple and intuitive terms, especially taking advantage of the independent-electron picture underlying DF.

The computations described in Sections 27.5 through 27.9 offer a wealth of information on the ground state structure of the broken-symmetry phase. At sufficiently low density $\left(r_{s} \geq 40\right)$, electron localization is complete and the ground state is a collection of charge droplets, each corresponding to one electron and $1 / 2$ Bohr magneton. The broken-symmetry state is an insulator, and therefore the transition could be detected by measurements of the low-frequency electric conductivity. Other general spectroscopic properties such as the Raman spectrum might also be significantly affected by the transition that is also likely to change the frequency, strength, and dispersion relation of plasmon excitations.

The distribution of the charge droplets is fairly regular, and defines a lattice whose structure is intermediate between bcc and fcc. Nevertheless, several defects are distributed in the lowest energy structures found by our numerical minimization. Disorder is apparent also in the spin distribution, which shows only a weak preference for antiferromagnetic coupling. Either positional and spin disorder might give rise to nonlinear features in the conductivity and in the Raman excitation spectrum of low-density wires.

Both spontaneous spin polarization and electron localization found in our computations have a counterpart in the results of recent experiments (Rahman and Sanyal 2007, Deshpande and Bockrath 2008, Danneau et al. 2008). Noncollinear spin states, excluded by our simple LSD scheme, might instead appear as broken-symmetry solutions both in real systems and in more sophisticated determinations of the nanowire electronic structure. In this respect, the complex density distribution found by our energy minimization at $r_{\mathrm{s}}=100, \zeta=0$ provides clear evidence that intriguing surprises may still be expected even from the simplest jellium model of nanowires.

\section{References}

E. Arrigoni (1999). Crossover from Luttinger- to Fermi-liquid behavior in strongly anisotropic systems in large dimensions. Phys. Rev. Lett. 83:128.

E. Arrigoni (2000). Crossover to Fermi-liquid behavior for weakly coupled Luttinger liquids in the anisotropic largedimension limit. Phys. Rev. B 61:7909.

N. W. Ashcroft and N. D. Mermin (1976). Solid State Physics. Holt-Saunders, London, U.K.

O. M. Auslaender et al. (2002). Tunneling spectroscopy of the elementary excitations in a one-dimensional wire. Science 295:825.

O. M. Auslaender et al. (2005). Spin-charge separation and localization in one dimension. Science 308:88.

J. M. Baik et al. (2008). Nanostructure-dependent metal-insulator transitions in vanadium-oxide nanowires. Phys. Chem. C, Lett. 112:13328.

R. Beckman et al. (2005). Bridging dimensions: Demultiplexing ultrahigh-density nanowire circuits. Science 310:465.

S. Biermann et al. (2001). Deconfinement transition and Luttinger liquid to Fermi liquid cross over in quasi-one-dimensional systems. Phys. Rev. Lett. 87:276405.

S. Biermann et al. (2002). Quasi one-dimensional organic conductors: Dimensional crossover and some puzzles. In: Strongly Correlated Fermions and Bosons in Low-Dimensional Disordered Systems. Kluwer Academic Publishers, Dordrecht, the Netherlands.

I. M. L. Billas et al. (1993). Magnetic moments of iron clusters with 25 to 700 atoms and their dependence on temperature. Phys. Rev. Lett. 71:4067.

J. P. Bird and Y. Ochiai (2004). Electron spin polarizations in nanoscale constrictions. Nature 303:1621.

M. Bockrath et al. (1999). Luttinger-liquid behaviour in carbon nanotubes. Nature 397:598.

M. Brack (1993). The physics of simple metal clusters: Selfconsistent jellium model and semiclassical approaches. Rev. Mod. Phys. 65:677.

J. Cao et al. (2005). Electron transport in very clean, As-grown suspended carbon nanotubes. Nature 4:745.

M. Casula et al. (2006). Ground state properties of the onedimensional Coulomb gas using the lattice regularized diffusion Monte Carlo method. Phys. Rev. B 74:245427.

D. M. Ceperley and B. J. Alder (1980). Ground state of the electron gas by a stochastic method. Phys. Rev. Lett. 45:566.

J. C. Charlier et al. (2007). Electronic and transport properties of nanotubes. Rev. Mod. Phys. 79:677.

R. Cortes-Huerto et al. (2009). To be published. 
Y. Cui and M. Lieber (2001). Functional nanoscale electronic devices assembled using silicon nanowire building blocks. Science 291:851.

R. Danneau et al. (2008). 0.7 structure and zero bias anomaly in ballistic hole quantum wires. Phys. Rev. Lett. 100:016403.

W. A. de Heer (1993). The physics of simple metal clusters: Experimental aspects and simple models. Rev. Mod. Phys. 65:611.

V. V. Deshpande and M. Bockrath (2008). The one-dimensional Wigner crystal in carbon nanotubes. Nat. Phys. 4:314.

N. D. Drummond et al. (2004). Diffusion quantum Monte-Carlo study of three-dimensional Wigner crystals. Phys. Rev. B 69:085116

H. J. Fan et al. (2006). Semiconductor nanowires: From self-organization to growth control. Small 2:700.

H. A. Fertig and L. Brey (2006). Luttinger liquid at the edge of undoped graphene in a strong magnetic field. Phys. Rev. Lett. 97:116805.

G. A. Fiete (2007). Colloquium: The spin-incoherent Luttinger liquid. Rev. Mod. Phys. 79:801.

A. V. Filinov et al. (2001). Wigner crystallization in mesoscopic 2D electron systems. Phys. Rev. Lett. 86:3851.

M. E. Fisher (1998). Renormalization group theory: Its basis and formulation in statistical physics. Rev. Mod. Phys. 70:653.

A. Georges et al. (2000). Interchain conductivity of coupled Luttinger liquids and organic conductors. Phys. Rev. B 61:16393.

G. F. Giuliani and G. Vignale (2005). Quantum Theory of the Electron Liquid, 1st edn. Cambridge University Press, Cambridge, U.K.

A. Gold and L. Calmels (1998). Excitation spectrum of the quasione-dimensional electron gas with long-range Coulomb interaction. Phys. Rev. B 58:3497.

N. W. Gong et al. (2008). $\mathrm{Au}\left(\mathrm{Si}\right.$ )-filled $\beta-\mathrm{Ga}_{2} \mathrm{O}_{3}$ nanotubes as wide range high temperature nanothermometers. Appl. Phys. Lett. 92:073101.

C. Grimes and G. Adams (1979). Evidence for a liquid-to-crystal phase transition in a classical, two-dimensional sheet of electrons. Phys. Rev. Lett. 42:795.

H. Hasegawa et al. (2008). Organic Mott insulator-based nanowire formation by using the nanoscale-electrocrystallization. Thin Solid Films 516:2491.

T. Hertel et al. (1998). Manipulation of individual carbon nanotubes and their interaction with surfaces. J. Phys. Chem. B 102:910.

K. Hiraki and K. Kanoda (1998). Wigner crystal type of charge ordering in an organic conductor with a quarter-filled band: (DI-DCNQI) ${ }_{2}$ Ag. Phys. Rev. Lett. 80:4737.

P. Hohenberg and W. Kohn (1964). Inhomogenous electron gas. Phys. Rev. 136:B864.

P. Horsch et al. (2005). Wigner crystallisation in $\mathrm{Na}_{3} \mathrm{Cu}_{2} \mathrm{O}_{4}$ and $\mathrm{Na}_{8} \mathrm{Cu}_{5} \mathrm{O}_{10}$ chain compounds. Phys. Rev. Lett. 94:076403.

D. Hughes and P. Ballone (2008). Spontaneous spin polarization and electron localization in constrained geometries: The Wigner transition in nanowires. Phys. Rev. B 77:245312-245322.
J. C. Inkson (1984). Many Body Theory of Solids. Plenum, New York.

Y. Ito and Y. Teraoka (2004). Magnetic phase diagram in a three-dimensional electron gas. J. Magn. Magn. Mater. 272-276S:E273.

Y. Ito and Y. Teraoka (2006). Wigner film ground state in a three-dimensional electron gas. J. Magn. Magn. Mater. 310:1073-1075.

M. José-Yacamán et al. (1993). Catalytic growth of carbon microtubules with fullerene structure. Appl. Phys. Lett. 62:657.

A. Kawabata (2007). Electron conduction in one-dimension. Rep. Prog. Phys. 70:219.

S. H. Kim et al. (2007). Cesium-filled single wall carbon nanotubes as conducting nanowires: Scanning tunneling spectroscopy study. Phys. Rev. Lett. 99:256407.

W. Kohn and L. J. Sham (1965). Self-consistent equations including exchange and correlation. Phys. Rev. 140:1133.

G. Kotliar and D. Vollhardt (2004). Strongly correlated materials: Insights from dynamical mean-field theory. Phys. Today 53.

G. Kotliar et al. (2006). Electronic structure calculations with dynamical mean-field theory. Rev. Mod. Phys. 78:865.

L. Kuipers and J. W. M. Frenken (1993). Jump to contact, neck formation, and surface melting in the scanning tunneling microscope. Phys. Rev. Lett. 70:3907.

R. Landauer (1970). Electrical resistance of disordered onedimensional lattices. Philos. Mag. 21:863.

Z. Lenac (2005). Interaction of the electromagnetic field with a Wigner crystal. Phys. Rev. B 71:035330.

C. Loo et al. (2004). Nanoshell-enabled photonics-based imaging and therapy of cancer. Technol. Cancer Res. Treat. 3:33.

J. M. Luttinger (1963). An exactly soluble model of a many-fermion system. J. Math. Phys. 4:1154.

G. D. Mahan (2000). Many-Particle Physics, 3rd edn. Kluwer Academic/Plenum Publishers, New York

R. M. Martin (2004). Electronic Structure. Basic Theory and Practical Methods, 1st edn. Cambridge University Press, Cambridge, U.K.

D. Marx and J. Hutter (2000). Ab Initio Molecular Dynamics: Theory and Implementation, vol. 1 of NIC Series. FZ Jülich, Berlin, Germany.

J. S. Meyer and K. A. Matveev (2009). Wigner crystal physics in quantum wires. J. Phys. Condens. Matter 21:023203.

M. J. Montgomery et al. (2002). Power dissipation in nanoscale conductors. J. Phys.: Condens. Matter 14:5377.

T. Morimoto et al. (2003). Nonlocal resonant interaction between coupled quantum wires. Appl. Phys. Lett. 82:3952.

E. J. Mueller (2005). Wigner crystallisation in inhomogeneous one-dimensional wires. Phys. Rev. B 72:075322.

J. W. Negele and H. Orland (1998). Quantum Many-Particle Systems. Addison-Wesley, Reading, MA.

J. C. Nickel et al. (2006). Superconducting pairing and densitywave instabilities in quasi-one-dimensional conductors. Phys. Rev. B 73:165126. 
M. Ogata and H. Fukuyama (1994). Collapse of quantized conductance in a dirty Tomonaga-Luttinger liquid'. Phys. Rev. Lett. 73:468.

G. Ortiz et al. (1999). Zero temperature phases of the electron gas. Phys. Rev. Lett. 82:5317.

R. V. Parthasarathy and C. R. Martin (1994). Template-synthesised polyaniline microtubules. Chem. Mater. 6:1627.

J. P. Perdew et al. (1990). Stabilized jellium: Structureless pseudopotential model for the cohesive and surface properties of metals. Phys. Rev. B 42:11627.

L. Pfeiffer et al. (1990). Formation of a high quality two-dimensional electron gas on cleaved GaAs. Appl. Phys. Lett. 56:1697.

J. Pouget and S. Ravy (1997). X-ray evidence of charge density wave modulations in the magnetic phases of (TMTSF)(2) PF6 and (TMTTF)(2)Br. Synth. Met. 85:1523.

A. Rahman and M. K. Sanyal (2007). Observation of charge density wave characteristics in conducting polymer nanowires: Possibility of Wigner crystallization. Phys. Rev. B 76:045110.

A. Rahman et al. (2006). Evidence of a ratchet effect in nanowires of a conducting polymer. Phys. Rev. B 73:125313.

D. J. Reilly et al. (2002). Density-dependent spin polarization in ultra-low-disorder quantum wires. Phys. Rev. Lett. 89:246801.

M. C. Roco et al. (eds.) (2000). Nanotechnology Research Directions: Vision for Nanotechnology Research and Development in the Next Decade. Springer, Heidelberg, Germany.

K. I. Schuller et al. (2008). Keynote Lecture at the "Trends in Nanotechnology" Conference. Oviedo, Spain. 1-5 September, Abstract:www.tntconf.org/2008/Files/Abstracts/Keywords/ TNT2008_Schuller.pdf.

H. J. Schulz (1993). Wigner crystal in one-dimension. Phys. Rev. Lett. 71:1864.

H. J. Schulz et al. (2000). Fermi liquids and Luttinger liquids. Field Theories for Low-Dimensional Condensed Matter Systems. Springer, New York. (arXiv.org:cond-mat/9807366).

G. Senatore and G. Pastore (1990). Density-functional theory of freezing for quantum systems: The Wigner crystallization. Phys. Rev. Lett. 64:303.

L. Shulenburger et al. (2008). Correlation effects in quasi-onedimensional quantum wires. Phys. Rev. B 78:165303.

C. A. Stafford et al. (1997). Jellium model of metallic nanocohesion. Phys. Rev. Lett. 79:2863.

H. Steinberg et al. (2006). Localization transition in a ballistic quantum wire. Phys. Rev. B 73.

S. Tanaka and S. Ichimaru (1987). Dynamic theory of correlations in strongly coupled, classical one-component plasmas: Glass transition in the generalized viscoelastic formalism. Phys. Rev. A 35:4743.

B. Tanatar et al. (1998). Wigner crystallization in semiconductor quantum wires. Phys. Rev. B 58:9886.

K. J. Thomas et al. (1996). Possible spin polarization in a onedimensional electron gas. Phys. Rev. Lett. 77:135.
S. Tomonaga (1950). Remarks on Bloch's method of sound waves applied to many-fermion problems. Prog. Theor. Phys. 5:544.

J. R. Trail et al. (2003). Unrestricted Hartree-Fock theory of Wigner crystals. Phys. Rev. B 68:045107.

J. M. van Ruitenbeek et al. (1997). Conductance quantization in metals: The influence of subband formation on the relative stability of specific contact diameters. Phys. Rev. B 56:12566.

B. J. van Wees et al. (1988). Quantized conductance of point contacts in a two-dimensional electron gas. Phys. Rev. Lett. 60:848.

P. Werner et al. (2006). On the formation of Si-nanowires by molecular beam epitaxy. Int. J. Mater. Res. 97:1008.

E. Wigner (1934). On the interaction of electrons in metals. Phys. Rev. 46:1002.

J. A. Yan et al. (2007). Size and orientation dependence in the electronic properties of silicon nanowires. Phys. Rev. B 76:115319.

L. Yang et al. (2007). Enhanced electron-hole interaction and optical absorption in a silicon nanowire. Phys. Rev. B. 75:201304.

C. Yannouleas and U. Landman (1997). On mesoscopic forces and quantised conductance in model metallic nanowires. J. Phys. Chem. B 101:5780.

C. Yannouleas et al. (1998). Energetics, forces, and quantized conductance in jellium-modeled metallic nanowires. Phys. Rev. B 57:4872.

S. N. Yanushkevich and B. Steinbach (2008). Spatial interconnect analysis for predictable nanotechnologies. J. Comp. Theor. Nanosci. 5:56.

Y. Yoon et al. (2007). Probing the microscopic structure of bound states in quantum point contacts. Phys. Rev. Lett. 99:136805.

W. S. Yun et al. (2000). Fabrication of metal nanowire using carbon nanotube as a mask. J. Vac. Sci. Technol. A 18:1329.

N. Zabala et al. (1998). Spontaneous magnetization of simple metal nanowires. Phys. Rev. Lett. 80:3336.

N. Zabala et al. (1999). Electronic structure of cylindrical simplemetal nanowires in the stabilized jellium model. Phys. Rev. B 59:12652.

S. V. Zaitsev-Zotov et al. (2000). Lutinger-liquid-like transport in long InSb nanowires. J. Phys. Condens. Matt. 12:L303.

Z. Zanolli et al. (2007). Quantum-confinement effects in InAs-InP core-shell nanowires. J. Phys. Condens. Matt. 19:295219.

X. Zhao et al. (2004). Quantum confinement and electronic properties of Silicon nanowires. Phys. Rev. Lett. 92:236805.

B. Zhou et al. (eds.) (2003). Nanotechnology in Catalysis, Vols. 1 and 2 (Nanostructure Science and Technology). Springer, New York.

F. H. Zong et al. (2002). Spin polarisation of the low-density threedimensional electron gas. Phys. Rev. E 66:036703. 


\section{Author Queries}

[AQ1] Please check whether the source line of figures 27.5 and 27.6 is correct.

[AQ2] Figure 27.15 to be set in grayscale. Please rephrase references to color in the figure caption appropriately. [AQ3] " $\alpha$ " is not shown in Equation 27.16.

[AQ4] Figure 27.20 to be set in grayscale. Please rephrase references to color in the figure caption appropriately.

[AQ5] Please check the sentence: “The approximation....caution." for sense.

[AQ6] Please update the reference Cortes-Huerto et al. (2009). 